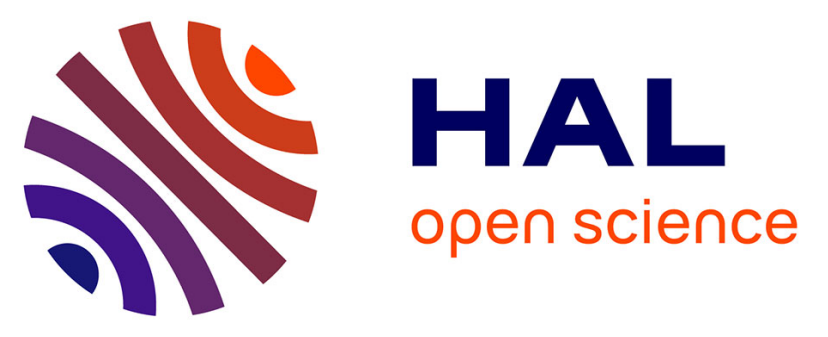

\title{
Synthesis of S-Trifluoromethyl S-Arylsulfoximine Thioglycosides through Pd-Catalyzed Migita Cross-Coupling
}

Nedjwa Bennai, Nada Ibrahim, Jérôme Marrot, Mohamed Belkadi, Mouad Alami, Emmanuel Magnier, Elsa Anselmi, Samir Messaoudi

\section{To cite this version:}

Nedjwa Bennai, Nada Ibrahim, Jérôme Marrot, Mohamed Belkadi, Mouad Alami, et al.. Synthesis of S-Trifluoromethyl S-Arylsulfoximine Thioglycosides through Pd-Catalyzed Migita Cross-Coupling. European Journal of Organic Chemistry, 2020, 10.1002/ejoc.202000821 . hal-02992809

\section{HAL Id: hal-02992809 https://hal.science/hal-02992809}

Submitted on 6 Nov 2020

HAL is a multi-disciplinary open access archive for the deposit and dissemination of scientific research documents, whether they are published or not. The documents may come from teaching and research institutions in France or abroad, or from public or private research centers.
L'archive ouverte pluridisciplinaire HAL, est destinée au dépôt et à la diffusion de documents scientifiques de niveau recherche, publiés ou non, émanant des établissements d'enseignement et de recherche français ou étrangers, des laboratoires publics ou privés. 


\title{
Synthesis of S-Trifluoromethyl S-Arylsulfoximine Thioglycosides through Pd-Catalyzed Migita Cross-Couling
}

\author{
Nedjwa Bennai, ${ }^{a, b}$ Nada Ibrahim, ${ }^{a}$ Jérôme Marrot, ${ }^{c}$ Mohamed Belkadi, ${ }^{b}$ Mouad Alami, ${ }^{a}$ Emmanuel \\ Magnier, ${ }^{* c}$ Elsa Anselmi, ${ }^{c, d}$ and Samir Messaoudia* \\ Dedication ((optional))
}
[a] N. Bennai, Drs. N. Ibrahim, M. Alami, S. Mesaoudi
Université Paris-Saclay, CNRS, BioCIS, 92290, Châtenay-Malabry, France
E-mail: samir.messaoudi@universite-paris-saclay.fr
[b] N. Bennai, Dr. M. Belkadi
Université des sciences et de la technologie d'Oran-Mohamed-Boudiaf, Algeria
[c] J. Marrot, Drs. E. Magnier, E. Anselmi
Université Paris-Saclay, UVSQ, CNRS, UMR 8180, Institut Lavoisier de Versailles, 78000, Versailles, France 2
[d] Dr. E. Anselmi
Université de Tours, Faculté des Sciences et Techniques, 37200 Tours, France
Supporting information for this article is given via a link at the end of the document.((Please delete this text if not appropriate))

\begin{abstract}
A general protocol for the synthesis of S-trifluoromethyl Sarylsulfoximine thioglycosides has been reported. This protocol is based on a Pd-catalyzed Migita cross-coupling between o-iodo Strifluoromethyl $\mathrm{S}$-arylsulfoximines and a broad range of 1-thiosugars. This method gives access to a series of functionalized S-trifluoromethyl S-arylsulfoximine thioglycosides in moderate to good yields. Moreover, both diastereoisomers were easily separated by simple crystallization or by HPLC and full characterizations are provided for each pure trifluoromethylsulfoximine.
\end{abstract}

\section{Introduction}

After a long period of latency, the sulfoximines have recently emerged as active pharmaceutical ingredients (APIs) in life and in crop sciences. This very peculiar group slowly evolved from "neglected opportunity in medicinal drug discovery" to an emergent and promising moiety. ${ }^{[1]}$ Some relevant examples are given as follows. BAY 1000394, a potent pan-CDK inhibitor and AZD 6738, an orally bioavailable inhibitor of the serine/threonine protein kinase ataxia telangiectasia mutated (ATM) are currently in phase II of clinical trials (Figure 1). ${ }^{[2]}$ An insecticide including a sulfoximine in its skeleton, the Sulfoxaflor ${ }^{\circledR}$ was launched on the market in 2013. ${ }^{[3]}$ In this context, the presence of fluorine atoms in a molecule dramatically changes its properties and in a vast majority of cases improves its efficiency for bioactive compounds purposes. ${ }^{[4]}$ Despite the importance of this halogen, S-perfluoroalkyl sulfoximines are extremely rare in the chemistry of life sciences whereas they are more and more present in the toolbox of the chemists as reagent in organic chemistry. ${ }^{[5]}$ One can hypothesize that fluorinated sulfoximines could bring original properties for relevant biological targets. A recent example highlighted indeed the interest of S-trifluoromethyl sulfoximine for antidiabetic activity (Figure 1). ${ }^{[6]}$ A library of pyrazines has been tested for antidiabetic properties. The presence of the fluorinated sulfoximine group proved efficient to increase cytosolic glucokinase levels through the binding to glucokinase regulatory protein (GRKP).

In another hand, 1-thioglycosides have emerged as a privileged class of glycosides with a diverse range of potential applications. ${ }^{[7]}$ These glycomimetics are known to be more

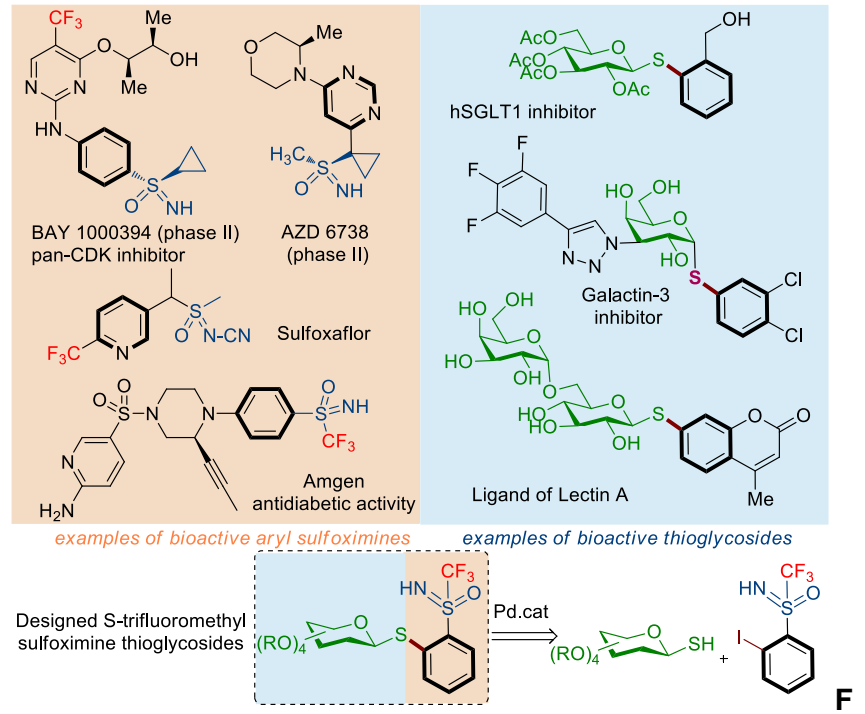

Figure 1. Biologically Active Aryl sulfoximines and Thioglycosides

resistant to glycosidase cleavage under biologically media. ${ }^{[7 f, 8]}$ Selected examples of bioactive thioglycosides are disclosed in Figure 1 such as hSGLT1 inhibitor, ligand of lectin A as well as Galactin-3 inhibitors. Moreover, thioglycosides are very useful intermediates in carbohydrate chemistry as they are largely used in sequential glycosylation tactics for oligosaccharides synthesis. [9]

Despite the potential interest of both sulfoximines and thiosugars in medicinal chemistry there is no report, to the best of our knowledge, devoted to synthesis of S-aryl Strifluoromethylsulfoximine thioglycosides. The combination of both these moieties in a designed single structure may however lead to the creation of unique molecular architectures based glycosides of interest for medicinal chemists. They could furthermore serve as potent drug analogues (Figure 1). This assumption is based on the fact that sulfoximines were proposed as alcohol isosteres. ${ }^{[10]}$ To reach our synthetic goal, we planned an approach merging the knowledge of our both research groups: in the chemistry of S-trifluoromethyl sulfoximine ${ }^{[11]}$ and in the development of strategies devoted to the reactivity of thiosugars under transition metal-catalysis ${ }^{[12]}$ including Pd-catalyzed Migita ${ }^{[13]}$ cross-coupling. The synthesis of o-iodo S-aryl S-trifluoromethylsulfoximine and the 
subsequent ${ }^{[14]}$ selective functionalization of the ortho position, in the presence of the free $\mathrm{NH}$ sulfoximine was recently carried out. ${ }^{[15]}$ Another interest of the present study would rely on the chiral pool offered by the thiosugars derivatives. This natural and cheap source of chirality could generate an easy access to pure diastereoisomers of S-trifluoromethyl sulfoximines thioglycosides This is of particular interest because methods to prepare S-trifluoromethyl sulfoximine diastereoisomers in a pure fashion are rare. ${ }^{[16]}$

\section{Results and Discussion}

To initiate this study, we conducted the coupling of tetra- $O$ acetylated 1-thio- $\beta$-D-galactopyranose $\mathbf{1 a}$ with o-iodo Strifluoromethyl S-phenylsulfoximine $\mathbf{2 a}$ as a model study under various reaction conditions. Representative results from this study are summarized in Table 1 . The reaction of $\mathbf{1 a}$ (1.2 equiv) with 2a (1 equiv) was first investigated under our previously reported conditions using $5 \mathrm{~mol} \%$ of a $\mathrm{PdG}_{3}$.XantPhos ${ }^{[17]}$ precatalyst in the presence of $\mathrm{Et}_{3} \mathrm{~N}$ ( 1.5 equiv) as a base at room temperature for 12 hours (Table 1, entry 1). However, under these conditions, the desired sulfoximine thioglycoside $3 \mathbf{a}$ has never been observed. Increasing the amount of the catalyst from $5 \mathrm{~mol} \%$ to $20 \mathrm{~mol} \%$ did not reveal any significant reactivity. This result may be explained by the hindrance effect of the trifluoromethyl arylsulfoximine group which may hamper the oxidative addition of the $\mathrm{Pd}(0)$ to the $\mathrm{C}-\mathrm{I}$ bond. At this stage, we decided to increase the activation energy of this reaction by performing the cross-coupling at $60{ }^{\circ} \mathrm{C}$ instead of room temperature. Under these conditions, the desired thioglycoside 3a was isolated in a good $65 \%$ yield as a single $\beta$-anomer $\left(J_{1,2}=\right.$ $9 \mathrm{~Hz})$ in a 1:1 diastereomeric ratio. Pleasingly, the two diastereoisomers showed distinct properties in solution as the pure 3aa- $(R)$ diastereoisomer could be crystallized in $\mathrm{Et}_{2} \mathrm{O}$ and easily separated from its $3 \mathbf{a b}-(S)$ isomer. Its exact structure was elucidated by X-ray analysis (Scheme 1 ). Moreover, the 3ab-(S) was obtained as a pure product upon evaporation of the liquid layer.

Table 1. Optimization of the Coupling Reaction of Tetraacetylated $\beta$ thiogalactose $\mathbf{1 a}$ with ortho-iodo-trifluoromethyl-arylsulfoximines $\mathbf{2} \mathbf{a}^{\mathbf{a}}$

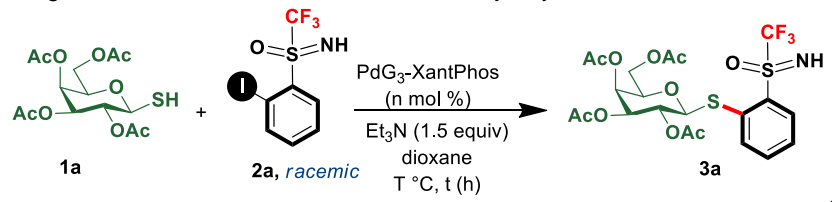

$\begin{array}{lllll}\text { entry } & \mathrm{n} \mathrm{mol} \% & \mathrm{~T}\left({ }^{\circ} \mathrm{C}\right) & \mathrm{T}(\mathrm{h}) & \mathbf{4 a}(\%)^{b} \\ 1 & 5 & \mathrm{rt} & 12 & 0 \\ 2 & 10 & \mathrm{rt} & 12 & 0 \\ 3 & 20 & \mathrm{rt} & 12 & 0 \\ 4 & 10 & 60 & 0.5 & 65 \\ 5 & 10 & 60 & 1 & 63 \\ 6 & 5 & 60 & 0.5 & 64\end{array}$

[a] A sealable tube was charged with thiosugar 1a (1.2 equiv, $0.2 \mathrm{mmol}$ ), ortho-iodo-trifluoromethyl-arylsulfoximines 2a (1 equiv), [Pd precatalyst] (n $\mathrm{mol} \%), \mathrm{Et}_{3} \mathrm{~N},(1.5$ equiv) in dioxane $(1.0 \mathrm{~mL})$. [b] Yield of isolated product.

It should be noted that the reaction is completely chemoselective and no by-product which could be arisen from the arylation of the nitrogen of the sulfoximine group was observed. Interesting, scale-up experiment $(1.5 \mathrm{mmol}$ of $\mathbf{2 a})$ was also performed with thiosugars $\mathbf{1 a}$, which led to the formation of thioglycoside $\mathbf{3 a}$ in an excellent $90 \%$ yield. Of note, the palladium catalyst is necessary to achieve this transformation since no reaction occurs when the coupling reaction is conducted in the absence of the catalyst.

With these results in hand, we subsequently investigated the substrate scope for this process with diverse mono-, di-, and trithiosugars 1b-j (Scheme 1). Gratifyingly, all the coupling reactions proceeded cleanly and selectively in good yields. This reaction is not limited to only $O$-acetylated 1 -thio- $\beta$-D-galactose 1a since different others sugars such as $\beta$-D-glucopyranose (1b) and $\beta$-D-fucose $(\mathbf{1 h})$ were used successfully providing $\mathbf{3 b}$ and

Scheme 1 Scope of Coupling of various Thiosugars $\mathbf{1 a - j}$ with $\mathbf{2 a - c ^ { a }}$
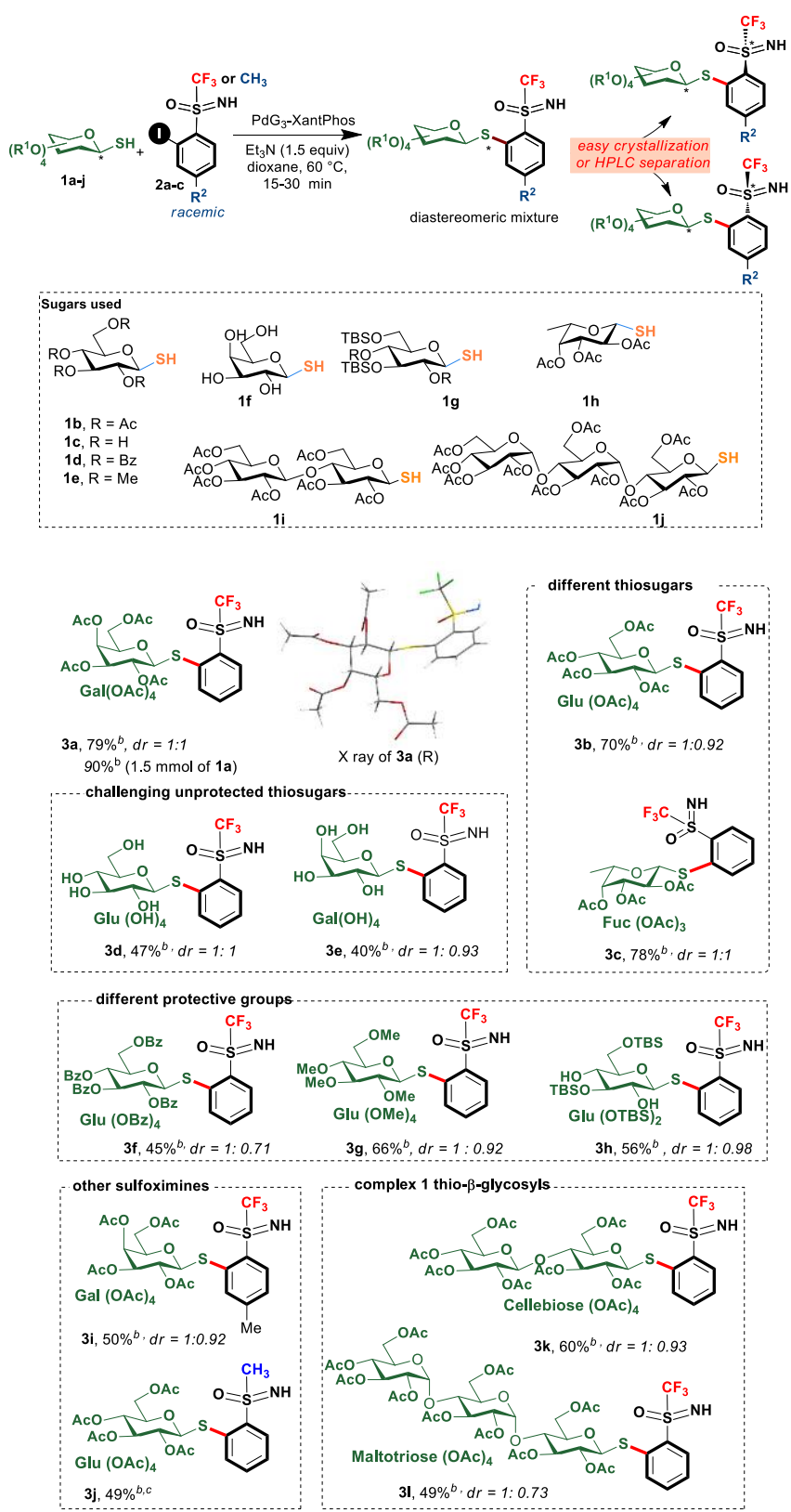

[a] Reaction conditions: A sealable tube was charged with thiosugar 1a-j (1.2 to 2 equiv), iodo-sulfoximine 2a-c (1 equiv) and XantPhos $\mathrm{PdG}_{3}$ precatalyst $(10 \mathrm{~mol} \%), \mathrm{Et}_{3} \mathrm{~N}$ (1.5 equiv) in dioxane. [b] Yield of isolated product. [c] 2 days reaction time 
3c in $70 \%$ and $78 \%$ yields, respectively. Unprotected thiosugars such as $\mathbf{1 c}$ and $\mathbf{1 f}$ can also serve as partners under our reaction conditions leading to the desired arylsulfoximine thioglycosides $\mathbf{3 d}$ and $\mathbf{3 e}$ in moderate yields. In addition, this methodology was not limited to only $O$-acetyl protected thiosugars but other protecting groups are tolerated such as $-\mathrm{OBz}(\mathbf{3 f}),-\mathrm{OMe}(\mathbf{3 g})$ and-OTBs (3h).

The structural variation of the aromatic partner was then investigated. The introduction of a methyl group on the aromatic ring was not deleterious to the transformation and the resulting compound $\mathbf{3 i}$ was isolated in $50 \%$ yield. However, although pure diastereosiomers of $\mathbf{3 i}$ were separated easily through HPLC purification and kept without alteration of the chiral information, epimerization of the chiral sulfoximine was observed in this only case indicating that the methyl group may alter the configurational stability of these diastereosiomers.

The same reaction with a non-fluorinated sulfoximine, a Smethyl analogue of $2 a$ was rather successful (3j isolated in $49 \%$ yield) but required an important increase of the reaction time, 2 days versus 30 minutes. This result illustrates the key role of the trifluoromethyl group in the general reactivity of the aromatic ring Next, we moved on to investigate the reactivity of complex and biologically relevant saccharide derivatives. Thus, we found that this coupling could be applied to thio- $\beta-D-c e l l o b i o s e ~ 1 i$ and thio$\beta$-D-maltotriose $\mathbf{1 j}$ delivering $\mathbf{3 k}$ and $\mathbf{3} \mathbf{I}$ in $60 \%$ and $49 \%$ yields, respectively.

Finally, we decided to investigate whether the chiral information on enantiopure trifluoromethyl sulfoximines was conserved during the cross-coupling process. In case of success, this may open the door to an efficient way to synthesize highly diastereopure trifluoromethyl sulfoximine thioglycosides. Delightfully, the reaction of enantiopure S-trifluoromethyl Sarylsulfoximines $^{16} \mathbf{2} \mathbf{a a}(R)$ and $2 \mathrm{ab}(S)$ under our optimized condition furnished the expected chiral products $\mathbf{3} \mathbf{a} \mathbf{a}^{\prime}(R)$ and 3ab' $(S)$ in $84 \%$ and $78 \%$ yields, respectively and without any detectable racemization by HPLC analysis or NMR (Scheme 2). To our knowledge, this result represent the first example of functionalization of enantiopure S-trifluoromethyl Sarylsulfoximines under Pd-catalysis, and may open a new way of diversifying the chemical space of S-trifluoromethyl S-arylsulfoximines through this methodology.

Scheme 2. Coupling of Chiral Sulfoximines 2aa and 2ab with thiogalactose $1 \mathbf{a}^{a}$<smiles>C=NS(=O)(=O)c1ccccc1C(F)(F)F</smiles>

2aa (R) enantiopur<smiles>O=S(=O)(NC(F)(F)F)c1ccccc1O</smiles>

2ab (S) enantiopur

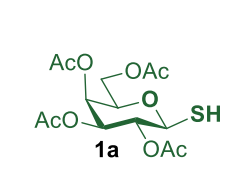

$$
\mathrm{PdG}_{3} \text {-XantPhos }
$$
$(10 \mathrm{~mol} \%)$

$$
\begin{gathered}
\mathrm{Et}_{3} \mathrm{~N}(1.5 \text { equiv) } \\
\text { dioxane } \\
60^{\circ} \mathrm{C}, 30 \mathrm{~min}
\end{gathered}
$$

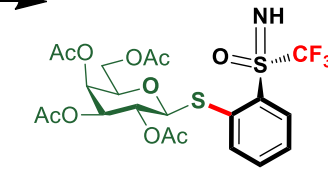

$3 a b^{\prime}, 78 \%^{b}(S)$
${ }^{a}$ Reaction conditions: A sealable tube was charged with thiosugar 1a (1.5 equiv, $0.2 \mathrm{mmol}$ ), iodo-sulfoximine $2 \mathrm{aa}$ or $2 \mathrm{ab}$ ( 1 equiv) and XantPhos $\mathrm{PdG}_{3}$ precatalyst (10 mol \%), $\mathrm{Et}_{3} \mathrm{~N}$ (1.5 equiv) in dioxane. ${ }^{b}$ Yield of isolated product.

\section{Conclusion}

In summary, we described here general protocol for the synthesis of S-trifluoromethyl S-arylsulfoximine thioglycosides. This protocol is based on a Pd-catalyzed Migita cross-coupling between o-iodo S-trifluoromethyl S-arylsulfoximines and a broad range of 1-thiosugars. Interestingly, the sugar part was used as a natural and cheap source of chirality that could generate an easy access to pure diastereomers of S-trifluoromethyl sulfoximines thioglycosides.

\section{Experimental Section}

Experimental Details.

\section{General information}

Solvents and reagents are obtained from commercial suppliers and were used without further purification. Analytical TLC was performed using Merck silica gel F254 (230-400 mesh) plates and analyzed by UV light or by staining upon heating with vanilin solution ( $15 \mathrm{~g}$ of vanilin in $250 \mathrm{~mL}$ ethanol and $2.5 \mathrm{~mL}$ of concentrated sulfuric acid). For silica gel chromatography, the flash chromatography technique was used, with Merck silica gel 60 (230-400 mesh) and p.a. grade solvents unless otherwise noted. The ${ }^{1} \mathrm{H}$ NMR and ${ }^{13} \mathrm{C}$ NMR spectra were recorded in either $\mathrm{CDCl}_{3}$ or $\mathrm{CD}_{3} \mathrm{OD}$ on Bruker Avance 300 spectrometers. NMR spectra for $19 \mathrm{~F}(188 \mathrm{MHz})$ were recorded in either $\mathrm{CDCl}_{3}$ or $\mathrm{CD}_{3} \mathrm{OD}$ on Bruker Avance 200 spectrometers. The chemical shifts of ${ }^{1} \mathrm{H},{ }^{13} \mathrm{C}$ and ${ }^{19} \mathrm{~F}$ are reported in ppm relative to the solvent residual peaks. IR spectra were measured on a Bruker Vector 22 spectrophotometer.Merck silica gel $60(0.015-0.040 \mathrm{~mm})$ was used for column chromatography. Melting points were recorded on a Büchi B-450 apparatus. High resolution mass spectra (HR-MS) were recorded on a MicroMass LCT Premier Spectrometer spectrometer. Optical rotations were obtained with a PolAAr 32 polarimeter. Thiosugars were synthesized according the literature protocols. ${ }^{[17]}$ 2-iodosulfoximines $2 \mathbf{2 a - c}$ were prepared as reported. ${ }^{[14]}$ The Xantphos Palladium precatalyst $\mathrm{PdG}_{3}$ was synthetized according to literature protocol. ${ }^{[18]}$

General procedure for the coupling of Thiosugars 1a-j with 2iodosulfoximine 2 a-c

A sealable tube purged with argon equipped with a cap was charged with $\mathrm{PdG}_{3}$-Xantphos (10 mol \%), B-thiosugar (1.2 to 2 equiv), 2-iodosulfoximine (1 equiv). Then, Dioxane $(1 \mathrm{~mL})$ was added and to the solution was added $\mathrm{Et}_{3} \mathrm{~N}$ (1.5 equiv) dropwise, and the mixture was stirred at $60^{\circ} \mathrm{C}$ for $30 \mathrm{~min}$. The reaction mixture was cooled to room temperature and passed through celite and rinsed with EtOAc. Then the filtrate was concentrated and the residue was purified by column chromatography on silica gel. The liquid chromatography separation of the diastereoisomers was achieved using an Agilent infinity 1260 (4.6x $150 \mathrm{~mm}) 5 \mu \mathrm{m}$ column.

\section{Compounds characterization}

(2R,3S,4S,5R,6S)-2-(acetoxymethyl)-6-((2-S(trifluoromethyl)sulfonimidoyl) phenyl)thio)tetrahydro-2H-pyran-3,4,5-triyl triacetate 3a

A sealable tube purged with argon equipped with a cap was charged with $\mathrm{PdG}_{3}$-Xantphos (28 mg, $0.03 \mathrm{mmol}, 10 \mathrm{~mol} \%$ ), tetraacetylated Bthiogalactose $1 \mathrm{a}$ (130 mg, $0.36 \mathrm{mmol}, 1.2$ equiv), 2-iodo-sulfoximine $2 \mathrm{a}$ (100 mg, $0.3 \mathrm{mmol}, 1$ equiv). Dioxane $(1 \mathrm{~mL}$ ) was added to the mixture and then, $\mathrm{Et}_{3} \mathrm{~N}(61 \mu \mathrm{L}, 0.45 \mathrm{mmol}, 1.5$ equiv) dropwise. The solution was stirred at $60^{\circ} \mathrm{C}$ for $30 \mathrm{~min}$. The reaction mixture was cooled to room 
temperature and passed through celite and rinsed with EtOAc. This crude was purified by column chromatography on silica gel (Cyclohexane/ EtOAc: 5/5), and 3a was isolated as a white solid in $65 \%$ yield. The ratio of diastereoisomers of compound $\mathbf{3 a}$ was $1: 1$ measured by NMR.

Protocol for crystallization and separation of $(R)$ and (S) diastereoisomers for compound 3a:

To a mixture of $(R)$ and $(S)$ diastereoisomers $(100 \mathrm{mg}$ ) was added $5 \mathrm{~mL}$ of diethyl ether and the solution was stored at $4^{\circ} \mathrm{C}$ for $24 \mathrm{~h}$. A white solid crystal was formed and isolated through simple filtration. The exact structure of the crystal was elucidated by X-ray analysis as the diastereoisomer 3aa- $(R)$. Additionally, the diastereoisomer 3ab- $(S)$ was obtained as a pure product upon evaporation of the liquid layer. This operation was repeated 2 to 3 times until the liquid layer delivered only a pure $3 a b-(S)$.

$(2 R, 3 S, 4 S, 5 R, 6 S)-2-($ acetoxymethyl)-6-((2-)-((R)-S-

(trifluoromethyl)sulfonimidoyl)phenyl)thio)tetrahydro-2H-pyran-3,4,5-triyl triacetate $3 a a$

$\mathbf{R}_{\mathbf{f}}(50 \%$ EtOAc/Pentane $)=0.57 ;[\mathbf{\alpha}]_{\mathbf{D}}{ }^{19}=+8.37$ (c, 1.66 in $\left.\mathrm{CHCl}_{3}\right) ; \mathbf{m p}$ $50.5-54.2^{\circ} \mathbf{C}$; IR (neat, $\mathbf{~ c m}^{-1}$ ) 1749, 1368, 1213, 1174, 1083, 1058; ${ }^{1} \mathbf{H}$ NMR $\left(300 \mathrm{MHz}, \mathrm{CDCl}_{3}\right) \delta 8.27(\mathrm{~d}, J=8.0 \mathrm{~Hz}, 1 \mathrm{H}), 7.92(\mathrm{~d}, J=8.0 \mathrm{~Hz}$, $1 \mathrm{H}), 7.65(\mathrm{t}, J=7.7 \mathrm{~Hz}, 1 \mathrm{H}), 7.49(\mathrm{t}, J=7.6 \mathrm{~Hz}, 1 \mathrm{H}), 5.48(\mathrm{~d}, J=3.1 \mathrm{~Hz}$, $1 \mathrm{H}), 5.41$ (t, $J=10.0 \mathrm{~Hz}, 1 \mathrm{H}), 5.10(\mathrm{dd}, J=9.9,3.3 \mathrm{~Hz}, 1 \mathrm{H}), 4.89(\mathrm{~d}, J=$ $9.9 \mathrm{~Hz}, 1 \mathrm{H}), 4.23-3.99(\mathrm{~m}, 3 \mathrm{H}), 3.95(\mathrm{bs}, \mathrm{NH}), 2.18(\mathrm{~s}, 3 \mathrm{H}), 2.05(\mathrm{~s}, 6 \mathrm{H})$ 1.99 (s, 3H); ${ }^{13} \mathrm{C}$ NMR $\left(75 \mathrm{MHz}, \mathrm{CDCl}_{3}\right) \delta 170.36\left(\mathrm{C}_{\mathrm{q}}\right), 170.16\left(\mathrm{C}_{\mathrm{q}}\right)$, $170.06\left(\mathrm{C}_{\mathrm{q}}\right), 169.39\left(\mathrm{C}_{\mathrm{q}}\right), 138.99\left(\mathrm{C}_{\mathrm{q}}\right), 135.22(\mathrm{CH}), 133.96(\mathrm{CH}), 132.45$ $(\mathrm{CH}), 131.83\left(\mathrm{C}_{\mathrm{q}}\right), 127.52(\mathrm{CH}), 121.16\left(\mathrm{q}, \mathrm{J}_{\mathrm{CF}}=333 \mathrm{~Hz}, \mathrm{CF}_{3}\right), 85.60$ $(\mathrm{CH}), 74.81(\mathrm{CH}), 72.08(\mathrm{CH}), 67.34(\mathrm{CH}), 66.67(\mathrm{CH}), 61.84\left(\mathrm{CH}_{2}\right)$, 20.74 et $20.63\left(4 \times \mathrm{CH}_{3}\right) ;{ }^{19} \mathrm{~F}$ NMR $\left(188 \mathrm{MHz}, \mathrm{CDCl}_{3}\right) \delta-76.55\left(\mathrm{CF}_{3}\right)$; HRMS(ESI): $\mathrm{m} / \mathrm{z}$ calcd for $\mathrm{C}_{21} \mathrm{H}_{25} \mathrm{~F}_{3} \mathrm{NO}_{10} \mathrm{~S}_{2}[\mathrm{M}+\mathrm{H}]+572.0866$; found 572.0866. Aspect: White crystal solid.

\section{(2R,3S, 4S,5R,6S)-2-(acetoxymethyl)-6-((2-)((S)-S-}

(trifluoromethyl)sulfonimidoyl)phenyl)thio)tetrahydro-2H-pyran-3,4,5-triyl triacetate $3 a b$

$\mathbf{R}_{\mathbf{f}}(50 \%$ EtOAc/Pentane $)=0.5 ;[\boldsymbol{\alpha}]_{\mathbf{D}}{ }^{19}=+83.43\left(\mathrm{c}, 1.66\right.$ in $\left.\mathrm{CHCl}_{3}\right) ; \mathbf{m p}$ $48.1-52.3^{\circ} \mathbf{C}$; IR (neat, $\mathbf{~ c m}^{-1}$ ) 1760, 1367, 1243, 1085, 1069; ${ }^{\mathbf{H}} \mathbf{H}$ NMR $\left(300 \mathrm{MHz}, \mathrm{CDCl}_{3}\right) \delta 8.19(\mathrm{~d}, J=8.0 \mathrm{~Hz}, 1 \mathrm{H}), 7.87(\mathrm{~d}, J=8.0 \mathrm{~Hz}, 1 \mathrm{H})$, $7.65(\mathrm{t}, J=7.7 \mathrm{~Hz}, 1 \mathrm{H}), 7.47(\mathrm{t}, J=7.7 \mathrm{~Hz}, 1 \mathrm{H}), 5.48(\mathrm{~d}, J=3.2 \mathrm{~Hz}, 1 \mathrm{H})$, 5.37 (t, $J=9.9 \mathrm{~Hz}, 1 \mathrm{H}$ ), 5.09 (dd, $J=9.9,3.2 \mathrm{~Hz}, 1 \mathrm{H}$ ), 4.81 (d, $J=10.1$ $\mathrm{Hz}, 1 \mathrm{H}), 4.26-4.03(\mathrm{~m}, 4 \mathrm{H}), 2.18(\mathrm{~s}, 3 \mathrm{H}), 2.07(\mathrm{~s}, 3 \mathrm{H}), 2.04(\mathrm{~s}, 3 \mathrm{H}), 1.99$ (s, 3H),; ${ }^{13} \mathrm{C}$ NMR (75 MHz, $\left.\mathbf{C D C l}_{3}\right) \delta 170.33\left(\mathrm{C}_{\mathrm{q}}\right), 170.24\left(\mathrm{C}_{\mathrm{q}}\right), 170.03$ $\left(\mathrm{C}_{\mathrm{q}}\right), 169.44\left(\mathrm{C}_{\mathrm{q}}\right), 140.70\left(\mathrm{C}_{\mathrm{q}}\right), 135.51(\mathrm{CH}), 133.42(\mathrm{CH}), 131.14(\mathrm{CH})$, $128.41\left(\mathrm{C}_{\mathrm{q}}\right), 127.07(\mathrm{CH}), 121.33\left(\mathrm{q}, \mathrm{J}_{\mathrm{CF}}=334 \mathrm{~Hz}, \mathrm{CF}_{3}\right), 86.49(\mathrm{CH})$, $74.63(\mathrm{CH}), 71.97(\mathrm{CH}), 67.26(\mathrm{CH}), 66.80(\mathrm{CH}), 61.99\left(\mathrm{CH}_{2}\right), 20.73$, 20.62 et $20.53\left(4 \times \mathrm{CH}_{3}\right) ;{ }^{19} \mathrm{~F}$ NMR $\left(188 \mathrm{MHz}, \mathrm{CDCl}_{3}\right)$ ठ $-76.25\left(\mathrm{CF}_{3}\right)$; HRMS(ESI): $\mathrm{m} / \mathrm{z}$ calcd for $\mathrm{C}_{21} \mathrm{H}_{25} \mathrm{~F}_{3} \mathrm{NO}_{10} \mathrm{~S}_{2}[\mathrm{M}+\mathrm{H}]+572.0866$; found 572.0864. Aspect : White solid.

(2R,3R,4S,5R,6S)-2-(acetoxymethyl)-6-((2-(S-

(trifluoromethyl)sulfonimidoyl)phenyl)thio)tetrahydro-2H-pyran-3,4,5-triyl triacetate $\mathbf{3 b}$

A sealable tube purged with argon equipped with a cap was charged with $\mathrm{PdG}_{3}$-Xantphos (28 mg, $0.03 \mathrm{mmol}, 10 \mathrm{~mol} \%$ ), tetraacetylated Bthioglucose $1 \mathrm{~b}$ (130 $\mathrm{mg}, 0.36 \mathrm{mmol}, 1.2$ equiv), 2-iodo-sulfoximine 2a (100 mg, $0.3 \mathrm{mmol}, 1$ equiv). Dioxane $\left(1 \mathrm{~mL}\right.$ ) was added followed by $\mathrm{Et}_{3} \mathrm{~N}$ $\left(61 \mu \mathrm{L}, 0.45 \mathrm{mmol}, 1.5\right.$ equiv) dropwise. The mixture was stirred at $60^{\circ} \mathrm{C}$ for $30 \mathrm{~min}$.

The reaction mixture was cooled to room temperature and passed through a plug of celite and rinsed with EtOAc. The crude was purified by column chromatography on silica gel (Cyclohexane/ EtOAc: 5/5), and compound $\mathbf{3 b}$ was isolated as a white solid in $70 \%$ yield. The ratio of diastereoisomers of compound $\mathbf{3 b}$ was about 1:0.91 (by NMR).

The chromatographic separation was achieved using an Agilent infinity $1260(4.6 \times 150 \mathrm{~mm}) 5 \mu \mathrm{m}$ column. The mobile phase was Acetonitrile and $\mathrm{H}_{2} \mathrm{O}$ in the ratio of $60: 40, \mathrm{v} / \mathrm{v}$ in $25 \mathrm{~min}$. The column temperature was maintained at $25^{\circ} \mathrm{C}$ and the eluent was monitored at a wavelength of 280 $\mathrm{nm}$. The injection volume was $10 \mu \mathrm{L}$. The typical retention time of diastereoisomers of compound $\mathbf{3 b}$ was about $19.1 \mathrm{~min}$ and $20.8 \mathrm{~min}$, respectively.

\section{Diastereoisomer 3ba}

$\mathbf{R}_{\mathbf{f}}(50 \%$ EtOAc/Cyclohexane $)=0.37 ;[\alpha]_{D^{19}}=-58.43\left(\mathrm{c}, 1.66\right.$ in $\left.\mathrm{CHCl}_{3}\right) ;$ mp: $145.2-147.5^{\circ} \mathbf{C}$; IR (neat, $\mathbf{c m}^{-1}$ ) 1752, 1452, 1368, 1252, 1034; ${ }^{\text {H }}$ NMR $\left(300 \mathrm{MHz}, \mathrm{CDCl}_{3}\right) \delta 8.26(\mathrm{~d}, J=7.9 \mathrm{~Hz}, 1 \mathrm{H}), 7.84(\mathrm{~d}, J=7.8 \mathrm{~Hz}$, $1 \mathrm{H}), 7.65(\mathrm{t}, J=7.4 \mathrm{~Hz}, 1 \mathrm{H}), 7.49(\mathrm{t}, J=7.4 \mathrm{~Hz}, 1 \mathrm{H}), 5.28(\mathrm{t}, J=9.1 \mathrm{~Hz}$, $1 \mathrm{H}), 5.19-5.07(\mathrm{~m}, 2 \mathrm{H}), 4.92(\mathrm{~d}, J=10.0 \mathrm{~Hz}, 1 \mathrm{H}), 4.80(\mathrm{bs}, \mathrm{NH}), 4.25-$ $4.15(\mathrm{~m}, 2 \mathrm{H}), 3.89-3.76(\mathrm{~m}, 1 \mathrm{H}), 2.08\left(\mathrm{~s}, \mathrm{CH}_{3}\right), 2.04\left(\mathrm{~s}, \mathrm{CH}_{3}\right), 2.03(\mathrm{~s}$, $\left.\mathrm{CH}_{3}\right)$ and $2.00\left(\mathrm{~s}, \mathrm{CH}_{3}\right), ;{ }^{13} \mathrm{C}$ NMR $\left(75 \mathbf{M H z}, \mathbf{C D C l}_{3}\right) \delta 170.52\left(\mathrm{C}_{\mathrm{q}}\right)$, $170.21\left(\mathrm{C}_{\mathrm{q}}\right), 169.47\left(\mathrm{C}_{\mathrm{q}}\right), 169.33\left(\mathrm{C}_{\mathrm{q}}\right), 138.57\left(\mathrm{C}_{\mathrm{q}}\right), 135.34(\mathrm{CH}), 133.98$ $(\mathrm{CH}), 132.67(\mathrm{CH}), 132.09\left(\mathrm{C}_{\mathrm{q}}\right), 127.72(\mathrm{CH}), 121.13\left(\mathrm{q}, \mathrm{J}_{\mathrm{CF}}=330 \mathrm{~Hz}\right.$, $\left.\mathrm{CF}_{3}\right), 85.13(\mathrm{CH}), 76.05(\mathrm{CH}), 73.96(\mathrm{CH}), 69.68(\mathrm{CH}), 68.33(\mathrm{CH}), 62.36$ $\left(\mathrm{CH}_{2}\right), 20.78\left(\mathrm{CH}_{3}\right), 20.64\left(2 \times \mathrm{CH}_{3}\right), 20.55\left(\mathrm{CH}_{3}\right) ;{ }^{19} \mathrm{~F}$ NMR (188 MHz, $\left.\mathrm{CDCl}_{3}\right) \delta-76.47\left(\mathrm{CF}_{3}\right)$; HRMS(ESI): $\mathrm{m} / \mathrm{z}$ calcd for $\mathrm{C}_{21} \mathrm{H}_{26} \mathrm{~F}_{3} \mathrm{NO}_{10} \mathrm{~S}_{2}$ $[\mathrm{M}+2 \mathrm{H}]+573.0939$; found 573.0997. Aspect: White solid.

\section{Diastereoisomer $3 b b$}

$\mathbf{R}_{\mathbf{f}}(50 \%$ EtOAc/Cyclohexane $)=0.33 ;[\alpha]_{\mathbf{D}}{ }^{19}=-68.85\left(\mathrm{c}, 1.66\right.$ in $\left.\mathrm{CHCl}_{3}\right) ;$ mp: $179.1-181.8^{\circ} \mathbf{C}$; IR (neat, $\mathbf{~ c m}^{-1}$ ) 1753, 1453, 1215, 1033, $737 ;{ }^{1} \mathbf{H}$ NMR $\left(300 \mathrm{MHz}, \mathrm{CDCl}_{3}\right) \delta 8.19(\mathrm{~d}, J=7.9 \mathrm{~Hz}, 1 \mathrm{H}), 7.84(\mathrm{~d}, J=8.0 \mathrm{~Hz}$, $1 \mathrm{H}), 7.65(\mathrm{t}, J=7.3 \mathrm{~Hz}, 1 \mathrm{H}), 7.48(\mathrm{t}, J=6.9 \mathrm{~Hz}, 1 \mathrm{H}), 5.28(\mathrm{t}, J=9.2 \mathrm{~Hz}$, $1 \mathrm{H}), 5.15-5.07(\mathrm{~m}, 2 \mathrm{H}), 4.82(\mathrm{~d}, J=10.1 \mathrm{~Hz}, 1 \mathrm{H}), 4.22(\mathrm{~m}, 2 \mathrm{H}), 3.92-$ $3.83(\mathrm{~m}, \mathrm{CH}+\mathrm{NH}), 2.10(\mathrm{~s}, 3 \mathrm{H}), 2.05-2.00(\mathrm{~m}, 9 \mathrm{H}) ;{ }^{13} \mathrm{C}$ NMR $(75 \mathrm{MHz}$, $\left.\mathrm{CDCl}_{3}\right)$ ठ $170.53\left(\mathrm{C}_{\mathrm{q}}\right), 170.20\left(\mathrm{C}_{\mathrm{q}}\right), 169.47\left(\mathrm{C}_{\mathrm{q}}\right), 169.34\left(\mathrm{C}_{\mathrm{q}}\right), 140.59\left(\mathrm{C}_{\mathrm{q}}\right)$, $135.56(\mathrm{CH}), 133.41(\mathrm{CH}), 132.71\left(\mathrm{C}_{\mathrm{q}}\right), 131.16(\mathrm{CH}), 127.17(\mathrm{CH})$, $121.34\left(\mathrm{q}, \mathrm{J}_{\mathrm{CF}}=332 \mathrm{~Hz}, \mathrm{CF}_{3}\right), 86.15(\mathrm{CH}), 75.89(\mathrm{CH}), 73.88(\mathrm{CH}), 69.76$ $(\mathrm{CH}), 68.39(\mathrm{CH}), 62.51\left(\mathrm{CH}_{2}\right), 20.81\left(\mathrm{CH}_{3}\right), 20.66\left(2 \times \mathrm{CH}_{3}\right), 20.46\left(\mathrm{CH}_{3}\right)$; ${ }^{19} \mathrm{~F}$ NMR (188 MHz, $\left.\mathrm{CDCl}_{3}\right)$ $\delta$-76.14 $\left(\mathrm{CF}_{3}\right) ; \mathrm{HRMS}(\mathrm{ESI}): \mathrm{m} / \mathrm{z}$ calcd for $\mathrm{C}_{21} \mathrm{H}_{26} \mathrm{~F}_{3} \mathrm{NO}_{10} \mathrm{~S}_{2}[\mathrm{M}+2 \mathrm{H}]+573.0939$; found 573.0997. Aspect : White solid.

(2S,3R,4R,5S,6R)-2-methyl-6-((2-(S-

(trifluoromethyl)sulfonimidoyl)phenyl)thio)tetrahydro-2H-pyran-3,4,5-triyl triacetate $3 c$

A sealable tube purged with argon equipped with a cap was charged with $\mathrm{PdG}_{3}$-Xantphos (31 mg, $\left.0.033 \mathrm{mmol}, 10 \mathrm{~mol} \%\right)$, triacetylated Bthiofucose $\mathbf{1 h}$ ( $200 \mathrm{mg}, 0.66 \mathrm{mmol}, 2.0$ equiv), 2-iodo-sulfoximine $\mathbf{2 a}$ (110 $\mathrm{mg}, 0.33 \mathrm{mmol}, 1$ equiv). Then, Dioxane $(1 \mathrm{~mL})$ was added and to the solution was added $\mathrm{Et}_{3} \mathrm{~N}(67 \mu \mathrm{L}, 0.5 \mathrm{mmol}, 1.5$ equiv) dropwise and the mixture was stirred at $60^{\circ} \mathrm{C}$ for $30 \mathrm{~min}$.

The reaction mixture was cooled to room temperature and passed through a plug of celite and rinsed with EtOAc. This crude was purified by column chromatography on silica gel (Cyclohexane/ EtOAc: 6/4), and compound $3 \mathbf{c}$ was isolated as a white solid in $78 \%$ yield. The ratio of diastereoisomers of compound $\mathbf{3 c}$ was about 0.93:1 (by NMR).

The chromatographic separation was achieved using a Chiralpak AD-H $(250 \times 10 \mathrm{~mm})$, hexane / ethanol $(50 / 50)$ as mobile phase, flow-rate $=5$ $\mathrm{mL} / \mathrm{min}$, UV detection at $254 \mathrm{~nm}$. Injections: 50 times $80 \mu \mathrm{L}$, every 6.2 minutes.

Diastereoisomer 3ca 
$\mathbf{R}_{\mathbf{f}}(50 \%$ EtOAc/Cyclohexane $)=0.47 ;{ }^{1} \mathbf{H}$ NMR $\left(\mathbf{3 0 0} \mathbf{~ M H z}, \mathbf{C D C l}_{3}\right) \boldsymbol{\delta} 8.25$ $(\mathrm{d}, J=8.0 \mathrm{~Hz}, 1 \mathrm{H}), 7.94(\mathrm{~d}, J=8.0 \mathrm{~Hz}, 1 \mathrm{H}), 7.65(\mathrm{t}, J=7.7 \mathrm{~Hz}, 1 \mathrm{H}), 7.47$ (t, $J=7.7 \mathrm{~Hz}, 1 \mathrm{H}), 5.39(\mathrm{t}, J=10.0 \mathrm{~Hz}, 1 \mathrm{H}), 5.31(\mathrm{~m}, 1 \mathrm{H}), 5.09(\mathrm{dd}, J=$ 9.9, 3.2 Hz, $1 \mathrm{H}$ ), $4.85(\mathrm{~d}, J=10.1 \mathrm{~Hz}, 1 \mathrm{H}), 3.91(\mathrm{q}, J=6.3 \mathrm{~Hz}, 1 \mathrm{H}), 2.25$ (s, 3H), $2.04(\mathrm{~s}, 3 \mathrm{H}), 1.98(\mathrm{~s}, 3 \mathrm{H}), 1.26(\mathrm{~d}, J=6.5 \mathrm{~Hz}, 3 \mathrm{H}) ;{ }^{13} \mathrm{C}$ NMR $(75$ $\left.\mathbf{M H z}, \mathbf{C D C l}_{3}\right) \delta 170.57\left(\mathrm{C}_{\mathrm{q}}\right), 170.17\left(\mathrm{C}_{\mathrm{q}}\right), 169.48\left(\mathrm{C}_{\mathrm{q}}\right), 139.25\left(\mathrm{C}_{\mathrm{q}}\right)$, $135.27(\mathrm{CH}), 133.93(\mathrm{CH}), 132.35(\mathrm{CH}), 131.72\left(\mathrm{C}_{\mathrm{q}}\right), 127.38(\mathrm{CH})$, 121.20 (q, J $J_{\mathrm{CF}}=332 \mathrm{~Hz}, \mathrm{CF}_{3}$ ), $85.36(\mathrm{CH}), 73.58(\mathrm{CH}), 72.57(\mathrm{CH}), 70.39$ $(\mathrm{CH}), 66.66(\mathrm{CH}), 20.90\left(\mathrm{CH}_{3}\right), 20.80\left(\mathrm{CH}_{3}\right), 20.72\left(\mathrm{CH}_{3}\right), 16.61\left(\mathrm{CH}_{3}\right)$; HRMS(ESI): $\mathrm{m} / \mathrm{z}$ calcd for $\mathrm{C}_{19} \mathrm{H}_{23} \mathrm{~F}_{3} \mathrm{NO}_{8} \mathrm{~S}_{2}[\mathrm{M}+\mathrm{H}]+514.0812$; found 514.0811. Aspect : amorphous.

\section{Diastereoisomer 3cb}

$\mathbf{R}_{\mathbf{f}}(50 \%$ EtOAc/Cyclohexane $)=0.40 ;{ }^{1} \mathbf{H}$ NMR $\left(300 \mathbf{~ M H z}, \mathbf{C D C l}_{3}\right) \boldsymbol{\delta} 8.20$ (d, $J=8.0 \mathrm{~Hz}, 1 \mathrm{H}$ ), 7.86 (d, $J=8.0 \mathrm{~Hz}, 1 \mathrm{H}), 7.67$ (t, $J=7.7 \mathrm{~Hz}, 1 \mathrm{H}), 7.47$ (t, $J=7.7 \mathrm{~Hz}, 1 \mathrm{H}), 5.39-5.32(\mathrm{~m}, 2 \mathrm{H}), 5.09(\mathrm{dd}, J=9.9,3.2 \mathrm{~Hz}, 1 \mathrm{H})$, $4.79(\mathrm{~d}, J=10.1 \mathrm{~Hz}, 1 \mathrm{H}), 4.23(\mathrm{bs}, \mathrm{NH}), 3.93(\mathrm{q}, J=6.5 \mathrm{~Hz}, 1 \mathrm{H}), 2.20$ (s $3 \mathrm{H}), 2.03(\mathrm{~s}, 3 \mathrm{H}), 1.99(\mathrm{~s}, 3 \mathrm{H}), 1.28(\mathrm{~d}, J=6.5 \mathrm{~Hz}, 3 \mathrm{H}) ;{ }^{13} \mathrm{C}$ NMR $(75$ $\left.\mathbf{M H z}, \mathbf{C D C l}_{3}\right) \delta 170.69\left(\mathrm{C}_{\mathrm{q}}\right), 170.17\left(\mathrm{C}_{\mathrm{q}}\right), 169.54\left(\mathrm{C}_{\mathrm{q}}\right), 140.64\left(\mathrm{C}_{\mathrm{q}}\right)$, $135.60(\mathrm{CH}), 133.44(\mathrm{CH}), 131.37(\mathrm{CH}), 127.11(\mathrm{CH}), 121.36\left(\mathrm{q}, J_{\mathrm{CF}}=\right.$ $\left.332 \mathrm{~Hz}, \mathrm{CF}_{3}\right), 86.45(\mathrm{CH}), 73.35(\mathrm{CH}), 72.44(\mathrm{CH}), 70.28(\mathrm{CH}), 66.89$ $(\mathrm{CH}), 20.80\left(\mathrm{CH}_{3}\right), 20.72\left(\mathrm{CH}_{3}\right), 20.64\left(\mathrm{CH}_{3}\right), 16.64\left(\mathrm{CH}_{3}\right)$, (one carbon (Cq) is missing); HRMS(ESI): $\mathrm{m} / \mathrm{z}$ calcd for $\mathrm{C}_{19} \mathrm{H}_{23} \mathrm{~F}_{3} \mathrm{NO}_{8} \mathrm{~S}_{2}[\mathrm{M}+\mathrm{H}]$ +514.0812 ; found 514.0811 . Aspect : amorphous.

imino(trifluoromethyl)(2-(((2S,3R,4S,5S,6R)-3,4,5-trihydroxy-6(hydroxymethyl)tetrahydro-2H-pyran-2-yl)thio)phenyl)-16-sulfanone $\mathbf{3 d}$

A sealable tube purged with argon equipped with a cap was charged with $\mathrm{PdG}_{3}$-Xantphos (32 mg, $\left.0.034 \mathrm{mmol}, 10 \mathrm{~mol} \%\right)$, B-thioglucose 1c $(80 \mathrm{mg}$ $0.41 \mathrm{mmol}, 1.2$ equiv), 2-iodo-sulfoximine $2 \mathrm{a}(114 \mathrm{mg}, 0.34 \mathrm{mmol}, 1$ equiv). Then, a mixture of dioxane $/ \mathrm{H}_{2} \mathrm{O}(1 \mathrm{~mL} / 0.5 \mathrm{~mL})$ was added and to the solution was added $\mathrm{Et}_{3} \mathrm{~N}(69 \mu \mathrm{L}, 0.51 \mathrm{mmol}, 1.5$ equiv) dropwise. The mixture was stirred at $60^{\circ} \mathrm{C}$ for $30 \mathrm{~min}$.

The reaction mixture was cooled to room temperature and passed through a plug of celite and rinsed with EtOAc. This crude was purified by column chromatography on silica gel (DCM/ $\mathrm{MeOH} 8 / 2)$, and the desired product 3d was isolated as a yellow oil in $47 \%$ yield. The ratio of diastereoisomers of compound $\mathbf{3 d}$ was about 0.97:1 (by NMR).

The chromatographic separation was achieved using a Agilent infinity $1260(4.6 \times 150 \mathrm{~mm}) 5 \mu \mathrm{m}$ column. The mobile phase was Methanol and $\mathrm{H}_{2} \mathrm{O}+0.1 \mathrm{DEA}$ (Grad $\mathrm{MeOH} 30$ to $80 \%$ ) in $15 \mathrm{~min}$. The column temperature was maintained at $25^{\circ} \mathrm{C}$ and the eluent was monitored at a wavelength of $254 \mathrm{~nm}$. The injection volume was $10 \mu \mathrm{L}$. The typical retention time of diastereoisomers of compound $\mathbf{3 d}$ was about $9.5 \mathrm{~min}$ and $11.5 \mathrm{~min}$, respectively.

\section{Diastereoisomer 3da}

$\mathbf{R}_{\mathbf{f}}(20 \% \mathrm{MeOH} / \mathrm{DCM})=0.6 ;[\boldsymbol{\alpha}]_{\mathbf{D}}{ }^{19}=-37.53\left(\mathrm{c}, 1.66\right.$ in $\left.\mathrm{CHCl}_{3}\right)$; IR (neat, $\left.\mathbf{c m}^{-1}\right)$ 1634, 1452, 1279, 1042, $991 ;{ }^{1} \mathbf{H}$ NMR (300 MHz, CD ${ }_{3}$ OD) $\delta 8.18$ $(\mathrm{d}, J=7.7 \mathrm{~Hz}, 1 \mathrm{H}), 8.04(\mathrm{~d}, 8.1 \mathrm{~Hz}, 1 \mathrm{H}), 7.75(\mathrm{t}, J=7.5 \mathrm{~Hz}, 1 \mathrm{H}), 7.52(\mathrm{t}$, $J=7.7 \mathrm{~Hz}, 1 \mathrm{H}), 4.76(\mathrm{~d}, J=9.6 \mathrm{~Hz}, 1 \mathrm{H}), 3.95(\mathrm{~d}, J=11.9 \mathrm{~Hz}, 1 \mathrm{H}), 3.78-$ $3.59(\mathrm{~m}, 1 \mathrm{H}), 3.53-3.38(\mathrm{~m}, 4 \mathrm{H})$; this sample was contaminated by an amount of diethylamine used during the HPLC purification: pics at 3.36 (q) and $1.31(\mathrm{t}),{ }^{13} \mathrm{C}$ NMR (75 MHz, CD $\left.{ }_{3} \mathrm{OD}\right) \delta 142.53\left(\mathrm{C}_{\mathrm{q}}\right), 136.61(\mathrm{CH})$, $134.53(\mathrm{CH}), 132.11(\mathrm{CH}), 131.64\left(\mathrm{C}_{\mathrm{q}}\right), 127.28(\mathrm{CH}), 88.20(\mathrm{CH}), 80.84$ $(\mathrm{CH}), 76.37(\mathrm{CH}), 70.86(\mathrm{CH}), 70.45(\mathrm{CH}), 62.74\left(\mathrm{CH}_{2}\right)$, (one carbon ($\left.\mathrm{CF}_{3}\right)$ is missing); ${ }^{19} \mathrm{~F}$ NMR (188 $\left.\mathbf{M H z}, \mathrm{CD}_{3} \mathrm{OD}\right) \delta$-78.58 $\left(\mathrm{CF}_{3}\right)$; HRMS(ESI): $\mathrm{m} / \mathrm{z}$ calcd for $\mathrm{C}_{13} \mathrm{H}_{17} \mathrm{~F}_{3} \mathrm{NO}_{6} \mathrm{~S}_{2}[\mathrm{M}+\mathrm{H}]+404.0444$; found 404.0460. Aspect : Colorless oil.

\section{Diastereoisomer 3db}

$\mathbf{R}_{\mathbf{f}}(20 \% \mathrm{MeOH} / \mathrm{DCM})=0.5 ;[\boldsymbol{\alpha}]_{\mathbf{D}^{19}}=-2.11\left(\mathrm{c}, 1.66\right.$ in $\left.\mathrm{CHCl}_{3}\right) ;$ IR (neat, $\left.\mathbf{c m}^{-1}\right)$ 1634, 1453, 1280, 1105, $1016 ;{ }^{1} \mathbf{H}$ NMR (300 MHz, CD CD $_{3}$ OD $\delta 8.23$ (d, $J=7.8 \mathrm{~Hz}, 1 \mathrm{H}), 7.97(\mathrm{~d}, 8.1 \mathrm{~Hz}, 1 \mathrm{H}), 7.77(\mathrm{t}, J=7.7 \mathrm{~Hz}, 1 \mathrm{H}), 7.52(\mathrm{t}$, $J=7.5 \mathrm{~Hz}, 1 \mathrm{H}), 4.90(\mathrm{~s}, 1 \mathrm{H}), 3.50(\mathrm{~d}, J=11.8 \mathrm{~Hz}, 1 \mathrm{H}), 3.43(\mathrm{dd}, J=12.1$ $5.8 \mathrm{~Hz}, 1 \mathrm{H}), 3.56-3.37(\mathrm{~m}, 4 \mathrm{H})$; this sample was contaminated by a small amount of diethylamine used during the HPLC purification: pics at 2.97 (q) and $1.29(\mathrm{t}), ;{ }^{13} \mathrm{C}$ NMR $\left(75 \mathrm{MHz}, \mathrm{CD}_{3} \mathrm{OD}\right) \delta 142.47\left(\mathrm{C}_{\mathrm{q}}\right), 136.62$ $(\mathrm{CH}), 134.12(\mathrm{CH}), 132.83(\mathrm{CH}), 131.98\left(\mathrm{C}_{\mathrm{q}}\right), 122.69\left(\mathrm{q}, \mathrm{J}_{\mathrm{CF}}=333 \mathrm{~Hz}\right.$ $\left.\mathrm{CF}_{3}\right), 88.88(\mathrm{CH}), 80.78(\mathrm{CH}), 76.25(\mathrm{CH}), 70.66(\mathrm{CH}), 70.42(\mathrm{CH}), 62.79$ $\left(\mathrm{CH}_{2}\right) ;{ }^{19} \mathrm{~F}$ NMR (188 MHz, CD $\left.{ }_{3} \mathrm{OD}\right) \delta-78.12\left(\mathrm{CF}_{3}\right) ; \mathrm{HRMS}(\mathrm{ESI}): \mathrm{m} / \mathrm{z}$ calcd for $\mathrm{C}_{13} \mathrm{H}_{17} \mathrm{~F}_{3} \mathrm{NO}_{6} \mathrm{~S}_{2}[\mathrm{M}+\mathrm{H}]+404.0444$; found 404.0460 . Aspect : Colorless oil.

(2R,3R,4S,5R,6S)-2-(hydroxymethyl)-6-((2--(-imino(11oxidanyl)(trifluoromethyl)-I5-sulfanyl)phenyl)thio)tetrahydro-2H-pyran3,4,5-triol $3 e$

A sealable tube purged with argon equipped with a cap was charged with PdG 3 -Xantphos (61 mg, $0.064 \mathrm{mmol}, 10 \mathrm{~mol} \%$ ), B-thiogalactose $1 \mathrm{f}$ (150 $\mathrm{mg}, 0.768 \mathrm{mmol}, 1.2$ equiv), 2-iodo-sulfoximine $2 \mathrm{a}$ (213 mg, $0.64 \mathrm{mmol}, 1$ equiv). Then, dioxane $/ \mathrm{H}_{2} \mathrm{O}(1 \mathrm{~mL} / 0.5 \mathrm{~mL})$ was added before adding $\mathrm{Et}_{3} \mathrm{~N}$ $\left(130 \mu \mathrm{L}, 0.95 \mathrm{mmol}, 1.5\right.$ equiv) dropwise. The mixture was stirred at $60^{\circ} \mathrm{C}$ for $30 \mathrm{~min}$.

The reaction mixture was cooled to room temperature and passed through a plug of celite and rinsed with EtOAc. This crude was purified by column chromatography on silica gel (DCM/ MeOH: 9/1), and the desired product 3e was isolated as a yellow oil in $40 \%$ yield. The ratio of diastereoisomers of compound $3 \mathbf{e}$ was about 1:0.93 (by NMR).

The chromatographic separation was achieved using a Agilent infinity $1260(4.6 \times 150 \mathrm{~mm}) 5 \mu \mathrm{m}$ column. The mobile phase was Methanol and $\mathrm{H}_{2} \mathrm{O}+0.1 \mathrm{AcF}$ (Grad MeOH 25 to $40 \%$ ) in 15min. The column temperature was maintained at $25^{\circ} \mathrm{C}$ and the eluent was monitored at a wavelength of $254 \mathrm{~nm}$. The injection volume was $10 \mu \mathrm{L}$. The typical retention time of diastereoisomers of compound $3 \mathrm{e}$ was about $8.9 \mathrm{~min}$ and $9.8 \mathrm{~min}$, respectively.

\section{Diastereoisomer 3ea}

$\mathbf{R}_{\mathbf{f}}(10 \% \mathrm{MeOH} / \mathrm{DCM})=0.03 ;[\mathbf{\alpha}]_{\mathbf{D}}{ }^{19}=+12.53\left(\mathrm{c}, 1.66\right.$ in $\left.\mathrm{CHCl}_{3}\right) ; \mathbf{I R}$ (neat, $\mathbf{~ c m}^{-1}$ ) 1648, 1632, 1279, 1196, $1060 ;{ }^{1} \mathbf{H}$ NMR (300 MHz, CD $_{3}$ OD) $\delta 8.24(\mathrm{~d}, J=8.1 \mathrm{~Hz}, 1 \mathrm{H}), 8.03(\mathrm{~d}, J=7.9 \mathrm{~Hz}, 1 \mathrm{H}), 7.77(\mathrm{t}, J=7.1 \mathrm{~Hz}$, $1 \mathrm{H}), 7.53(\mathrm{t}, J=7.4 \mathrm{~Hz}, 1 \mathrm{H}), 4.83(\mathrm{~m}, 1 \mathrm{H}), 3.99(\mathrm{~d}, J=3.0 \mathrm{~Hz}, 1 \mathrm{H}), 3.88-$ $3.73(\mathrm{~m}, 4 \mathrm{H}), 3.62(\mathrm{dd}, J=9.1,3.2 \mathrm{~Hz}, 1 \mathrm{H}) ;{ }^{13} \mathrm{C}$ NMR $\left(75 \mathrm{MHz}, \mathrm{CD}_{3} \mathrm{OD}\right)$ ठ $142.53\left(\mathrm{C}_{\mathrm{q}}\right), 136.61(\mathrm{CH}), 134.53(\mathrm{CH}), 132.11(\mathrm{CH}), 131.64\left(\mathrm{C}_{\mathrm{q}}\right)$, $127.28(\mathrm{CH}), 88.20(\mathrm{CH}), 80.84(\mathrm{CH}), 76.37(\mathrm{CH}), 70.86(\mathrm{CH}), 70.45$ $(\mathrm{CH}), 62.74\left(\mathrm{CH}_{2}\right)$ (one carbon $\left(-\mathrm{CF}_{3}\right)$ is missing); ${ }^{19} \mathrm{~F}$ NMR (188 $\mathrm{MHz}$, $\left.\mathrm{CD}_{3} \mathrm{OD}\right) \delta$-78.59 $\left(\mathrm{CF}_{3}\right) ; \mathrm{HRMS}(\mathrm{ESI}): \mathrm{m} / \mathrm{z}$ calcd for $\mathrm{C}_{13} \mathrm{H}_{17} \mathrm{~F}_{3} \mathrm{NO}_{6} \mathrm{~S}_{2}[\mathrm{M}+\mathrm{H}]$ +404.0444; found 404.0454. Aspect : Yellow oil.

\section{Diastereoisomer 3eb}

$\mathbf{R}_{\mathbf{f}}(10 \% \mathrm{MeOH} / \mathrm{DCM})=0 ;[\boldsymbol{\alpha}]_{\mathbf{D}}{ }^{19}=+47.95\left(\mathrm{c}, 1.66\right.$ in $\left.\mathrm{CHCl}_{3}\right) ;$ IR (neat, $\left.\mathbf{c m}^{-1}\right)$ 1634, 1625, 1182, 1052; ${ }^{1} \mathbf{H}$ NMR (300 MHz, CD $\left._{3} \mathrm{OD}\right) \delta 8.22(\mathrm{~d}, J$ $=7.9 \mathrm{~Hz}, 1 \mathrm{H}), 8.12(\mathrm{~d}, J=8.1 \mathrm{~Hz}, 1 \mathrm{H}), 7.79(\mathrm{t}, J=7.7 \mathrm{~Hz}, 1 \mathrm{H}), 7.55(\mathrm{t}, J$ $=7.7 \mathrm{~Hz}, 1 \mathrm{H}), 4.77(\mathrm{~d}, J=9.6 \mathrm{~Hz}, 1 \mathrm{H}), 4.00(\mathrm{~d}, J=2.9 \mathrm{~Hz}, 1 \mathrm{H}), 3.90-$ $3.71(\mathrm{~m}, 4 \mathrm{H}), 3.62(\mathrm{dd}, J=9.1,3.1 \mathrm{~Hz}, 1 \mathrm{H}) ;{ }^{13} \mathrm{C}$ NMR $\left(75 \mathrm{MHz}, \mathrm{CD}_{3} \mathrm{OD}\right)$ ठ $142.47\left(\mathrm{C}_{\mathrm{q}}\right), 136.62(\mathrm{CH}), 134.12(\mathrm{CH}), 132.83(\mathrm{CH}), 131.98\left(\mathrm{C}_{\mathrm{q}}\right)$, $127.40(\mathrm{CH}), 122.69\left(\mathrm{q}, \mathrm{J}_{\mathrm{CF}}=333 \mathrm{~Hz}, \mathrm{CF}_{3}\right), 88.88(\mathrm{CH}), 80.78(\mathrm{CH})$, $76.25(\mathrm{CH}), 70.66(\mathrm{CH}), 70.42(\mathrm{CH}), 62.79\left(\mathrm{CH}_{2}\right) ;{ }^{19} \mathrm{~F}$ NMR (188 MHz, $\mathrm{CD}_{3} \mathrm{OD}$ ) $\delta$-78.12 $\left(\mathrm{CF}_{3}\right) ; \mathrm{HRMS}(\mathrm{ESI}): \mathrm{m} / \mathrm{z}$ calcd for $\mathrm{C}_{13} \mathrm{H}_{17} \mathrm{~F}_{3} \mathrm{NO}_{6} \mathrm{~S}_{2}[\mathrm{M}+\mathrm{H}]$ +404.0444; found 404.0454. Aspect : Yellow oil.

$(2 R, 3 R, 4 S, 5 R, 6 S)-2-(($ benzoyloxy $)$ methyl)-6-((2-(S-

(trifluoromethyl)sulfonimidoyl)phenyl)thio)tetrahydro-2H-pyran-3,4,5-triyl tribenzoate $\mathbf{3 f}$ 
A sealable tube purged with argon equipped with a cap was charged with $\mathrm{PdG}_{3}$-Xantphos (52 mg, $0.054 \mathrm{mmol}, 10 \mathrm{~mol} \%$ ), tetrabenzoylated Bthioglucose $\mathbf{1 d}$ (400 $\mathrm{mg}, 0.653 \mathrm{mmol}, 1.2$ equiv), 2-iodo-sulfoximine $\mathbf{2 a}$ (182 $\mathrm{mg}, 0.54 \mathrm{mmol}, 1$ equiv). After, dioxane $(1 \mathrm{~mL})$ was added before adding $\mathrm{Et}_{3} \mathrm{~N}(110 \mu \mathrm{L}, 0.815 \mathrm{mmol}, 1.5$ equiv) dropwise. Then, the mixture was stirred at $60^{\circ} \mathrm{C}$ for $30 \mathrm{~min}$.

The reaction mixture was cooled to room temperature and passed through a plug of celite and rinsed with EtOAc. This crude was purified by column chromatography on silica gel (Cyclohexane/ EtOAc: 5/5), and compound $\mathbf{3 f}$ was isolated as a yellow oil in $45 \%$ yield. The ratio of diastereoisomers of compound $\mathbf{3 f}$ was about 0.71:1 (by NMR).

The chromatographic separation was achieved using a Agilent infinity $1260(4.6 \times 150 \mathrm{~mm}) 5 \mu \mathrm{m}$ column. The mobile phase was Acetonitrile and $\mathrm{H}_{2} \mathrm{O}+0.1 \mathrm{AcF}$ in the ratio of $35: 65, \mathrm{v} / \mathrm{v}$ in $40 \mathrm{~min}$. The column temperature was maintained at $25^{\circ} \mathrm{C}$ and the eluent was monitored at a wavelength of $254 \mathrm{~nm}$. The injection volume was $10 \mu \mathrm{L}$. The typical retention time of diastereoisomers of compound $\mathbf{3 f}$ was about $22.0 \mathrm{~min}$ and $22.9 \mathrm{~min}$, respectively.

\section{Diastereoisomer 3 fa}

$\mathbf{R}_{\mathbf{f}}(50 \%$ EtOAc/Cyclohexane $)=0.74 ;[\alpha]_{\mathbf{D}}{ }^{19}=+47.95\left(\mathrm{c}, 1.66\right.$ in $\left.\mathrm{CHCl}_{3}\right)$ IR (neat, cm ${ }^{-1}$ ) 1728, 1451, 1258, 1067, 1025, 800, $706 ; \mathbf{1} \mathbf{H}$ NMR (300 $\left.\mathbf{M H z}, \mathbf{C D C l}_{3}\right) \delta 8.18(\mathrm{~d}, J=7.9 \mathrm{~Hz}, 1 \mathrm{H}), 8.01(\mathrm{~d}, J=7.3 \mathrm{~Hz}, 2 \mathrm{H}), 7.94$ $7.88(\mathrm{~m}, 6 \mathrm{H}), 7.81(\mathrm{~d}, J=7.4 \mathrm{~Hz}, 2 \mathrm{H}), 7.62-7.28\left(\mathrm{~m}, 13 \mathrm{H}+\right.$ pic of $\left.\mathrm{CHCl}_{3}\right)$, 5.99 (t, $J=9.4 \mathrm{~Hz}, 1 \mathrm{H}), 5.69-5.60(\mathrm{~m}, 2 \mathrm{H}), 5.27(\mathrm{~d}, J=10.0 \mathrm{~Hz}, 1 \mathrm{H}), 4.66$ (dd, $J=12.3,2.8 \mathrm{~Hz}, 1 \mathrm{H}), 4.51(\mathrm{dd}, J=12.1,6.6 \mathrm{~Hz}, 1 \mathrm{H}), 4.30-4.22(\mathrm{~m}$, $J=7.0 \mathrm{~Hz}, 1 \mathrm{H}), 3.72(\mathrm{bs}, \mathrm{NH}) ;{ }^{13} \mathbf{C}$ NMR (75 MHz, CDCl $\mathbf{~}_{3} \delta 165.89\left(\mathrm{C}_{\mathrm{q}}\right)$, $165.52\left(\mathrm{C}_{\mathrm{q}}\right), 165.23\left(\mathrm{C}_{\mathrm{q}}\right), 139.25\left(\mathrm{C}_{\mathrm{q}}\right), 135.28(\mathrm{CH}), 133.76(\mathrm{CH}), 133.47$ $(3 \times \mathrm{CH}), 130.05(\mathrm{CH}), 129.93(2 \times \mathrm{CH}), 128.83(6 \times \mathrm{CH}), 128.63\left(3 \times \mathrm{C}_{\mathrm{q}}\right)$, $128.49(4 \times \mathrm{CH}), 127.72(5 \times \mathrm{CH}), 85.74(\mathrm{CH}), 74.11(\mathrm{CH}), 70.48(\mathrm{CH})$ $69.63(\mathrm{CH}), 63.48\left(\mathrm{CH}_{2}\right)$, some carbons are missing; ${ }^{19} \mathrm{~F}$ NMR (188 $\mathbf{~ M H z}$, $\left.\mathrm{CDCl}_{3}\right)$ $\delta$-76.26 $\left(\mathrm{CF}_{3}\right)$; HRMS(ESI): $\mathrm{m} / \mathrm{z}$ calcd for $\mathrm{C}_{41} \mathrm{H}_{33} \mathrm{~F}_{3} \mathrm{NO}_{10} \mathrm{~S}_{2}[\mathrm{M}+\mathrm{H}]$ +820.1492 ; found 820.1474. Aspect : Yellow oil.

\section{Diastereoisomer 3fb}

$\mathbf{R}_{\mathbf{f}}(50 \%$ EtOAc/Cyclohexane $)=0.7 ;[\boldsymbol{\alpha}]_{\mathbf{D}}{ }^{19}=+35.48\left(\mathrm{c}, 1.66\right.$ in $\left.\mathrm{CHCl}_{3}\right) ;$ mp: 125.4 - $128.3{ }^{\circ} \mathbf{C}$; IR (neat, $\mathbf{c m}^{-1}$ ) 1721, 1244, 1177, 1063, 736, 705 ; ${ }^{1} \mathbf{H}$ NMR (300 MHz, $\left.\mathbf{C D C l}_{3}\right) \delta 8.08(\mathrm{t}, \mathrm{J}=8.5 \mathrm{~Hz}, 4 \mathrm{H}), 7.92(\mathrm{~m}, 6 \mathrm{H})$, $7.82(\mathrm{~d}, J=7.3 \mathrm{~Hz}, 2 \mathrm{H}), 7.60-7.18\left(\mathrm{~m}, 12 \mathrm{H}+\right.$ pic of $\left.\mathrm{CHCl}_{3}\right), 6.00(\mathrm{t}, J=$ $9.4 \mathrm{~Hz}, 1 \mathrm{H}), 5.74-5.62(\mathrm{~m}, 2 \mathrm{H}), 5.15(\mathrm{~d}, J=10.0 \mathrm{~Hz}, 1 \mathrm{H}), 4.73(\mathrm{~d}, J=$ $8.3 \mathrm{~Hz}, 1 \mathrm{H}), 4.58-4.50(\mathrm{~m}, 1 \mathrm{H}), 4.37-4.32(\mathrm{~m}, 1 \mathrm{H}), 4.02(\mathrm{bs}, \mathrm{NH}) ;{ }^{13} \mathrm{C}$ NMR (75 MHz, CDCl $)$ $) 166.09\left(\mathrm{C}_{\mathrm{q}}\right), 165.85\left(\mathrm{C}_{\mathrm{q}}\right), 165.43\left(\mathrm{C}_{\mathrm{q}}\right), 165.24$ $\left(\mathrm{C}_{\mathrm{q}}\right), 140.69\left(\mathrm{C}_{\mathrm{q}}\right), 135.51(\mathrm{CH}), 133.75(\mathrm{CH}), 133.49(2 \times \mathrm{CH}), 131.28(\mathrm{CH})$ $130.04(2 \times \mathrm{CH}), 129.90(6 \times \mathrm{CH}), 128.99\left(\mathrm{C}_{\mathrm{q}}\right), 128.79\left(\mathrm{C}_{\mathrm{q}}\right), 128.64(4 \times \mathrm{CH})$, $128.47(3 \times \mathrm{CH}), 128.36(2 \times \mathrm{CH}), 126.98(\mathrm{CH}), 120.97\left(\mathrm{q}, J_{\mathrm{CF}}=331 \mathrm{~Hz}\right.$, $\left.\mathrm{CF}_{3}\right), 86.64(\mathrm{CH}), 76.59(\mathrm{CH}), 74.04(\mathrm{CH}), 70.42(\mathrm{CH}), 69.66(\mathrm{CH}), 63.67$ $\left(\mathrm{CH}_{2}\right) ;{ }^{19} \mathrm{~F}$ NMR (188 MHz, $\left.\mathbf{C D C l}_{3}\right) \delta-76.22\left(\mathrm{CF}_{3}\right) ; \mathrm{HRMS}(\mathrm{ESI}): \mathrm{m} / \mathrm{z}$ calcd for $\mathrm{C}_{41} \mathrm{H}_{33} \mathrm{~F}_{3} \mathrm{NO}_{10} \mathrm{~S}_{2}[\mathrm{M}+\mathrm{H}]+820.1492$; found 820.1474. Aspect : Yellow solid.

(11-oxidanyl)(trifluoromethyl)(2-(((2S,3R,4S,5R,6R)-3,4,5-trimethoxy-6(methoxymethyl)tetrahydro-2H-pyran-2-yl)thio)phenyl)-I5-sulfanimine $\mathbf{3 g}$

A sealable tube purged with argon equipped with a cap was charged with $\mathrm{PdG}_{3}$-Xantphos (31 mg, $0.032 \mathrm{mmol}, 10 \mathrm{~mol} \%$ ), tetramethoxylated Bthioglucose $1 \mathrm{e}$ (160 $\mathrm{mg}, 0.634 \mathrm{mmol}, 2.0$ equiv), 2-iodo-sulfoximine $2 \mathrm{a}$ (106 mg, $0.32 \mathrm{mmol}, 1$ equiv). After, Dioxane $(1 \mathrm{~mL})$ was added before adding $\mathrm{Et}_{3} \mathrm{~N}$ (64 $\mu \mathrm{L}, 0.48 \mathrm{mmol}, 1.5$ equiv) dropwise. Then, the mixture was stirred at $60^{\circ} \mathrm{C}$ for $30 \mathrm{~min}$.

The reaction mixture was cooled to room temperature and passed through a plug of celite and rinsed with EtOAc. This crude was purified by column chromatography on silica gel (Cyclohexane/ EtOAc: 5/5), and compound $\mathbf{3 g}$ was isolated as a yellow oil in $66 \%$ yield. The ratio diastereoisomers of compound $\mathbf{3} \mathbf{g}$ was about 1:0.92 (by NMR).

The chromatographic separation was achieved using a Agilent infinity $1260(4.6 \times 150 \mathrm{~mm}) 5 \mu \mathrm{m}$ column. The mobile phase was Methanol and $\mathrm{H}_{2} \mathrm{O}+0.1 \mathrm{AcF}$ in the ratio of $45: 55, \mathrm{v} / \mathrm{v}$ in $40 \mathrm{~min}$. The column temperature was maintained at $25^{\circ} \mathrm{C}$ and the eluent was monitored at a wavelength of $254 \mathrm{~nm}$. The injection volume was $10 \mu \mathrm{L}$. The typical retention time of diastereoisomers of compound $\mathbf{3 g}$ was about $10.8 \mathrm{~min}$ and $11.5 \mathrm{~min}$, respectively.

\section{Diastereoisomer 3ga}

$\mathbf{R}_{\mathbf{f}}(50 \%$ EtOAc/Cyclohexane $)=0.5 ;[\alpha]_{\mathbf{D}}{ }^{19}=+91.8\left(\mathrm{c}, 1.66\right.$ in $\left.\mathrm{CHCl}_{3}\right)$ IR (neat, $\mathbf{c m}^{-1}$ ) 1756, 1452, 1177, 1093, 1030, 820, 736 ; $\mathbf{1} \mathbf{H}$ NMR (300 $\left.\mathrm{MHz}, \mathrm{CD}_{3} \mathrm{OD}\right) \delta 8.21(\mathrm{~d}, J=8.0 \mathrm{~Hz}, 1 \mathrm{H}), 7.98(\mathrm{~d}, J=8.1 \mathrm{~Hz}, 1 \mathrm{H}), 7.79(\mathrm{t}$ $J=7.7 \mathrm{~Hz}, 1 \mathrm{H}), 7.55(\mathrm{t}, J=7.7 \mathrm{~Hz}, 1 \mathrm{H}), 4.78(\mathrm{~d}, J=9.9 \mathrm{~Hz}, 1 \mathrm{H}), 3.73(\mathrm{~s}$, $4 \mathrm{H}), 3.67(\mathrm{~s}, 3 \mathrm{H}), 3.61(\mathrm{~s}, 3 \mathrm{H}), 3.6-3.55(\mathrm{~m}, 1 \mathrm{H}), 3.46(\mathrm{~s}, 3 \mathrm{H}), 3.41$ $3.38(\mathrm{~m}, 1 \mathrm{H}), 3.38-3.34(\mathrm{~m}, 1 \mathrm{H}), 3.26-3.14(\mathrm{~m}, 2 \mathrm{H}) ;{ }^{13} \mathrm{C}$ NMR (75 MHz, $\left.\mathbf{C D C l}_{3}\right) \delta 141.18\left(\mathrm{C}_{\mathrm{q}}\right), 135.62(\mathrm{CH}), 133.15(\mathrm{CH}), 131.79(\mathrm{CH}), 131.46$ $\left(\mathrm{C}_{\mathrm{q}}\right), 126.77(\mathrm{CH}), 121.37\left(\mathrm{q}, \mathrm{J}_{\mathrm{CF}}=332 \mathrm{~Hz}, \mathrm{CF}_{3}\right), 88.75(\mathrm{CH}), 87.79(\mathrm{CH})$, $82.87(\mathrm{CH}), 79.41(\mathrm{CH}), 78.98(\mathrm{CH}), 71.60\left(\mathrm{CH}_{2}\right), 61.18\left(\mathrm{CH}_{3}\right), 61.11$ $\left(\mathrm{CH}_{3}\right), 60.68\left(\mathrm{CH}_{3}\right), 59.56\left(\mathrm{CH}_{3}\right) ;{ }^{19} \mathrm{~F}$ NMR (188 MHz, $\left.\mathbf{C D C l}_{3}\right) \delta-76.52$ (CF $)$; HRMS(ESI): $\mathrm{m} / \mathrm{z}$ calcd for $\mathrm{C}_{17} \mathrm{H}_{25} \mathrm{~F}_{3} \mathrm{NO}_{16} \mathrm{~S}_{2}[\mathrm{M}+\mathrm{H}]+460.1069$; found 460.1068. Aspect : Colorless oil.

\section{Diastereoisomer $3 g b$}

$\mathbf{R}_{\mathbf{f}}(50 \%$ EtOAc/Cyclohexane $)=0.48 ;[\boldsymbol{\alpha}]_{\mathbf{D}}{ }^{19}=-2.11$ (c, 1.66 in $\left.\mathrm{CHCl}_{3}\right)$; IR (neat, $\mathbf{c m}^{-1}$ ) 1453, 1289, 1177, 1089, 1014, 797; ${ }^{\mathbf{H}} \mathbf{H}$ NMR (300 MHz, $\left.\mathrm{CDCl}_{3}\right) \delta 8.19(\mathrm{~d}, J=8.1 \mathrm{~Hz}, 1 \mathrm{H}), 8.04(\mathrm{~d}, J=8.2 \mathrm{~Hz}, 1 \mathrm{H}), 7.62(\mathrm{t}, J=$ $7.7 \mathrm{~Hz}, 1 \mathrm{H}), 7.40(\mathrm{t}, J=7.7 \mathrm{~Hz}, 1 \mathrm{H}), 5.80(\mathrm{~d}, J=5.5 \mathrm{~Hz}, 1 \mathrm{H}), 4.10-4.04$ (m, 2H), $3.65(\mathrm{~s}, 3 \mathrm{H}), 3.60$ (dd, J = 9.0 Hz, J=3.6 Hz, 1H), 3.55 (s, 3H), $3.46-3.39(\mathrm{~m}, 8 \mathrm{H}), 3.33-3.27(\mathrm{~m}, 1 \mathrm{H}) ;{ }^{13} \mathbf{C ~ N M R}\left(75 \mathbf{~ M H z} \mathbf{C D C l}_{3}\right) \delta$ $142.10\left(\mathrm{C}_{\mathrm{q}}\right), 135.48(\mathrm{CH}), 133.59(\mathrm{CH}), 131.15(\mathrm{CH}), 130.51\left(\mathrm{C}_{\mathrm{q}}\right), 126.06$ $(\mathrm{CH}), 85.61(\mathrm{CH}), 83.80(\mathrm{CH}), 81.58(\mathrm{CH}), 78.94(\mathrm{CH}), 71.55(\mathrm{CH}), 70.96$ $\left(\mathrm{CH}_{2}\right), 61.17\left(\mathrm{CH}_{3}\right), 60.60\left(\mathrm{CH}_{3}\right), 59.29\left(\mathrm{CH}_{3}\right), 57.89\left(\mathrm{CH}_{3}\right)$ (one carbon ($\left.\mathrm{CF}_{3}\right)$ is missing); ${ }^{19} \mathrm{~F}$ NMR (188 $\left.\mathbf{M H z} \mathbf{C D C l}_{3}\right) \delta-76.16\left(\mathrm{CF}_{3}\right)$; HRMS(ESI): $\mathrm{m} / \mathrm{z}$ calcd for $\mathrm{C}_{17} \mathrm{H}_{25} \mathrm{~F}_{3} \mathrm{NO}_{16} \mathrm{~S}_{2}[\mathrm{M}+\mathrm{H}]+460.1069$; found 460.1068. Aspect : Colorless oil.

(2-(((2S,3R,4S,5R,6R)-4-((tert-butyldimethylsilyl)oxy)-6-(((tertbutyldimethylsilyl)oxy)methyl)-3,5-dihydroxytetrahydro-2H-pyran-2yl)thio)phenyl)(imino)(trifluoromethyl)-l6-sulfanone $\mathbf{3 h}$

A sealable tube purged with argon equipped with a cap was charged with $\mathrm{PdG}_{3}$-Xantphos (7 mg, $\left.0.0075 \mathrm{mmol}, 10 \mathrm{~mol} \%\right), 2,4-$ tertbutyldimethylsilyl ß-thioglucose $\mathbf{1 g}(64 \mathrm{mg}, 0.1516 \mathrm{mmol}, 2.0$ equiv), 2iodo-sulfoximine 2a (25 mg, $0.075 \mathrm{mmol}, 1$ equiv). After, dioxane (1mL) was added before adding $\mathrm{Et}_{3} \mathrm{~N}(15 \mu \mathrm{L}, 0.112 \mathrm{mmol}, 1.5$ equiv) dropwise. Then, the mixture was stirred at $60^{\circ} \mathrm{C}$ for $30 \mathrm{~min}$.

The reaction mixture was cooled to room temperature and passed through a plug of celite and rinsed with EtOAc. This crude was purified by column chromatography on silica gel (DCM/ MeOH: $2 / 8)$, and compound 3h was isolated as a yellow oil in $56 \%$ yield. The ratio of diastereoisomers of compound $\mathbf{3 h}$ was about 1:0.98 (by NMR).

The diastereoisomers were separated directly by column chromatography on silica gel (DCM/MeOH : 2/8).

\section{Diastereoisomer 3ha}

$\mathbf{R}_{\mathbf{f}}(20 \% \mathrm{MeOH} / \mathrm{DCM})=0.53 ;[\boldsymbol{\alpha}]_{\mathbf{D}}{ }^{19}=-41.74$ (c, 1.66 in $\left.\mathrm{CHCl}_{3}\right) ;$ IR (neat, $\mathbf{c m}^{-1}$ ) 1250, 1076, 1005, 835, 811, 737; ${ }^{1} \mathbf{H}$ NMR (300 MHz, $\left.\mathrm{CDCl}_{3}\right) \delta 8.29(\mathrm{~d}, J=8.2 \mathrm{~Hz}, 1 \mathrm{H}), 7.95(\mathrm{~d}, J=8.0 \mathrm{~Hz}, 1 \mathrm{H}), 7.63$ (t, $J=$ $7.6 \mathrm{~Hz}, 1 \mathrm{H}), 7.48(\mathrm{t}, J=7.7 \mathrm{~Hz}, 1 \mathrm{H}), 4.62(\mathrm{~d}, J=9.6 \mathrm{~Hz}, 1 \mathrm{H}), 4.17-4.08$ 
$(\mathrm{m}, 2 \mathrm{H}), 3.94(\mathrm{dd}, J=11.2,2.6 \mathrm{~Hz}, 1 \mathrm{H}), 3.85(\mathrm{dd}, J=10.7,4.7 \mathrm{~Hz}, 1 \mathrm{H})$, $3.55-3.40(\mathrm{~m}, 3 \mathrm{H}), 2.92(\mathrm{~d}, J=2.4 \mathrm{~Hz}, 1 \mathrm{H}), 2.51(\mathrm{~s}, 1 \mathrm{H}), 0.90(\mathrm{~s}, 18 \mathrm{H})$, $0.14(\mathrm{~s}, 3 \mathrm{H}), 0.07(\mathrm{~s}, 9 \mathrm{H}) ;{ }^{13} \mathrm{C}$ NMR $\left(75 \mathrm{MHz}, \mathrm{CDCl}_{3}\right) \delta 135.46(\mathrm{CH})$, $135.35(\mathrm{CH}), 133.25(\mathrm{CH}), 127.99(\mathrm{CH}), 88.41(\mathrm{CH}), 79.83(\mathrm{CH}), 79.52$ $(\mathrm{CH}), 72.40(\mathrm{CH}), 71.59(\mathrm{CH}), 63.71\left(\mathrm{CH}_{2}\right), 29.85\left(2 \times \mathrm{C}_{\mathrm{q}}\right), 26.13\left(3 \times \mathrm{CH}_{3}\right)$, $26.08\left(3 \times \mathrm{CH}_{3}\right), 1.17\left(\mathrm{CH}_{3}\right),-4.00\left(\mathrm{CH}_{3}\right),-4.61\left(\mathrm{CH}_{3}\right),-5.2\left(\mathrm{CH}_{3}\right), 2$ Cqarom $+\mathrm{CF}_{3}$ carbons are missing; ${ }^{19} \mathrm{~F}$ NMR $\left(188 \mathrm{MHz}, \mathrm{CDCl}_{3}\right) \delta-76.61\left(\mathrm{CF}_{3}\right)$; HRMS(ESI): $\mathrm{m} / \mathrm{z}$ calcd for $\mathrm{C}_{25} \mathrm{H}_{45} \mathrm{~F}_{3} \mathrm{NO}_{6} \mathrm{~S}_{2} \mathrm{Si}_{2}[\mathrm{M}+\mathrm{H}]+632.2173$; found 632.2181. Aspect : Yellow oil.

\section{Diastereoisomer 3hb}

$\mathbf{R}_{\mathbf{f}}(20 \% \mathrm{MeOH} / \mathrm{DCM})=0.45 ;[\mathbf{\alpha}]_{D^{19}}=-56.32\left(\mathrm{c}, 1.66\right.$ in $\left.\mathrm{CHCl}_{3}\right) ; \quad$ IR (neat, $\mathbf{c m}^{-1}$ ) 1258, 1043, 1017, 835, 780, 737 ; ${ }^{1} \mathbf{H}$ NMR (300 MHz, $\left.\mathrm{CDCl}_{3}\right) \delta 8.25(\mathrm{~d}, J=8.0 \mathrm{~Hz}, 1 \mathrm{H}), 8.07(\mathrm{~d}, J=7.7 \mathrm{~Hz}, 1 \mathrm{H}), 7.64(\mathrm{t}, J=$ $7.6 \mathrm{~Hz}, 1 \mathrm{H}$ ), 7.52 (t, $J=7.6 \mathrm{~Hz}, 1 \mathrm{H}), 4.54(\mathrm{~d}, J=9.6 \mathrm{~Hz}, 1 \mathrm{H}), 4.17-4.07$ (m, 2H), 3.96 (dd, $J=10.8,2.1 \mathrm{~Hz}, 1 \mathrm{H}), 3.87(\mathrm{dd}, J=10.8,4.2 \mathrm{~Hz}, 1 \mathrm{H})$, $3.72-3.67(\mathrm{~m}, 1 \mathrm{H}), 3.57-3.40(3,2 \mathrm{H}), 3.25(\mathrm{dt}, J=9.9,2.1 \mathrm{~Hz}, 1 \mathrm{H}), 2.48$ (s, 1H), $0.93(\mathrm{~s}, 9 \mathrm{H}), 0.89(\mathrm{~s}, 9 \mathrm{H}), 0.18-0.05(\mathrm{~m}, 12 \mathrm{H}) ;{ }^{13} \mathrm{C}$ NMR $(75$ $\left.\mathbf{M H z}, \mathrm{CDCl}_{3}\right) \delta 137.90(\mathrm{Cq}), 135.47(\mathrm{CH}), 135.34(\mathrm{CH}), 133.24(\mathrm{CH})$, $132.3(\mathrm{Cq}), 127.99(\mathrm{CH}), 88.41(\mathrm{CH}), 79.83(\mathrm{CH}), 79.52(\mathrm{CH}), 72.39$ $(\mathrm{CH}), 71.59(\mathrm{CH}), 63.71\left(\mathrm{CH}_{2}\right), 29.86\left(2 \times \mathrm{C}_{\mathrm{q}}\right), 26.13\left(3 \times \mathrm{CH}_{3}\right), 26.08$ $\left(3 \times \mathrm{CH}_{3}\right), 1.17\left(\mathrm{CH}_{3}\right),-4.00\left(\mathrm{CH}_{3}\right),-4.61\left(\mathrm{CH}_{3}\right),-5.19\left(\mathrm{CH}_{3}\right), 1 \mathrm{Cq}\left(-\mathrm{CF}_{3}\right)$ carbons are missing; ${ }^{19} \mathrm{~F}$ NMR $\left(188 \mathrm{MHz}, \mathrm{CDCl}_{3}\right) \delta-76.18\left(\mathrm{CF}_{3}\right)$; HRMS(ESI): $\mathrm{m} / \mathrm{z}$ calcd for $\mathrm{C}_{25} \mathrm{H}_{45} \mathrm{~F}_{3} \mathrm{NO}_{6} \mathrm{~S}_{2} \mathrm{Si}_{2}[\mathrm{M}+\mathrm{H}]+632.2173$; found 632.2181. Aspect : Yellow oil.

\section{(2R,3S,4S,5R,6S)-2-(acetoxymethyl)-6-((5-methyl-2-(S-}

(trifluoromethyl)sulfonimidoyl)phenyl)thio)tetrahydro-2H-pyran-3,4,5-triyl triacetate $\mathbf{3 i}$

A sealable tube purged with argon equipped with a cap was charged with $\mathrm{PdG}_{3}$-Xantphos (23 mg, $0.024 \mathrm{mmol}, 10 \mathrm{~mol} \%$ ), tetraacetylated Bthiogalactose 1a (125 mg, $0.343 \mathrm{mmol}, 1.5$ equiv), 2-iodo,4-methylsulfoximine 2b $\left(80 \mathrm{mg}, 0.24 \mathrm{mmol}, 1\right.$ equiv). After, $\mathrm{Et}_{3} \mathrm{~N}(48 \mu \mathrm{L}, 0.36$ $\mathrm{mmol}, 1.5$ equiv) was added drop by drop. Then, Dioxane $(1 \mathrm{~mL})$ was added and the mixture was stirred at $60^{\circ} \mathrm{C}$ for $30 \mathrm{~min}$.

The reaction mixture was cooled to room temperature and passed through a plug of celite and rinsed with EtOAc. This coupling product was purified by column chromatography on silica gel (Pentane/ EtOAc: 6/4), and was isolated as a white solid in $50 \%$ yield. The ratio of diastereoisomers of compound $\mathbf{3 i}$ was about 1:1 (by NMR).

The chromatographic separation was achieved using a Chromatographic conditions: Lux-Cellulose-1 (250 x $10 \mathrm{~mm})$, hexane / ethanol (70/30) as mobile phase, flow-rate $=5 \mathrm{~mL} / \mathrm{min}$, UV detection at $254 \mathrm{~nm}$. Injections: 60 times $50 \mu \mathrm{L}$, every 4 minutes.

After the separation of the two diastereoisomers, we observed unfortunately epimerization during the storage. Herein we provided only NMR data of a mixture.

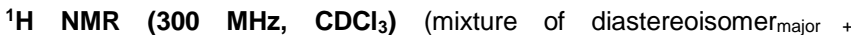
diastereoisomer $\left._{\text {minor }}\right) \delta 8.13\left(\mathrm{~d}, J=8.2 \mathrm{~Hz}, 1 \mathrm{H}_{\text {major }}, 8.06(\mathrm{~d}, J=9.0 \mathrm{~Hz}\right.$, $\left.1 \mathrm{H}_{\text {minor }}\right), 7.69\left(\mathrm{~s}, 1 \mathrm{H}_{\text {major }}\right), 7.65\left(\mathrm{~s}, 1 \mathrm{H}_{\text {minor }}\right), 7.27\left(\mathrm{~d}, J=8.2 \mathrm{~Hz}, 1 \mathrm{H}_{\text {majort }}\right.$

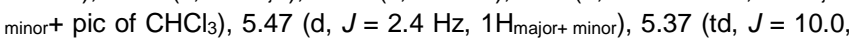
$7.1 \mathrm{~Hz}, 1 \mathrm{H}_{\text {major+ minor) }}, 5.10$ (dd, $J=9.9,3.3 \mathrm{~Hz}, 1 \mathrm{H}_{\text {major }}$ minor), 4.89 (d, $J=$ $10.1 \mathrm{~Hz}, 1 \mathrm{H}_{\text {major }}$ ), 4.83 (d, $J=10.1 \mathrm{~Hz}, 1 \mathrm{H}_{\text {minor }}$ ), 4.19-3.90 (m, 3H $\mathrm{H}_{\text {major }}$ minor

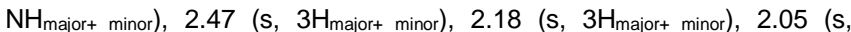

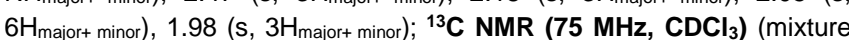
of diastereoisomer major + diastereoisomer $\left.{ }_{\text {minor }}\right) \delta 170.42\left(\mathrm{C}_{\mathrm{q}}\right), 170.22\left(\mathrm{C}_{\mathrm{q}}\right)$, $170.07\left(\mathrm{C}_{\mathrm{q}}\right), 169.42\left(\mathrm{C}_{\mathrm{q}}\right), 147.03\left(\mathrm{C}_{\mathrm{q}}\right), 146.79\left(\mathrm{C}_{\mathrm{q}}\right), 138.54\left(\mathrm{C}_{\mathrm{q}}\right), 134.05$ $\left(\mathrm{CH}_{\text {major }}\right), 133.56\left(\mathrm{CH}_{\text {minor }}\right), 133.32\left(\mathrm{CH}_{\text {major }}\right), 132.06\left(\mathrm{CH}_{\text {minor }}\right), 128.41$ $\left(\mathrm{CH}_{\text {major }}\right), 127.98\left(\mathrm{CH}_{\text {minor }}\right), 86.71\left(\mathrm{CH}_{\text {minor }}\right), 85.88\left(\mathrm{CH}_{\text {major }}\right), 74.89(\mathrm{CH})$, $74.71(\mathrm{CH}), 72.09(\mathrm{CH}), 72.01(\mathrm{CH}), 67.47(\mathrm{CH}), 66.82(\mathrm{CH}), 66.72(\mathrm{CH})$, $62.34\left(\mathrm{CH}_{2 \text { minor }}\right), 62.10$ ( $\left.\mathrm{CH}_{2 \text { major }}\right), 21.98\left(\mathrm{CH}_{3}\right) 20.78\left(2 \times \mathrm{CH}_{3}\right), 20.66$ $\left(2 \times \mathrm{CH}_{3}\right), 1 \mathrm{Cq}\left(-\mathrm{CF}_{3}\right)$ carbons are missing; HRMS(ESI): $\mathrm{m} / \mathrm{z}$ calcd for $\mathrm{C}_{22} \mathrm{H}_{27} \mathrm{~F}_{3} \mathrm{NO}_{10} \mathrm{~S}_{2}[\mathrm{M}+\mathrm{H}]+586.1023$; found 586.1102 . Aspect : White solid.

(2R,3R,4S,5R,6S)-2-(acetoxymethyl)-6-((2-(S-

methylsulfonimidoyl)phenyl)thio)tetrahydro-2H-pyran-3,4,5-triyl triacetate 3j

A sealable tube purged with argon equipped with a cap was charged with $\mathrm{PdG}_{3}$-Xantphos (34 mg, $0.036 \mathrm{mmol}, 10 \mathrm{~mol} \%$ ), tetraacetylated Bthioglucose $1 \mathrm{~b}$ (156 mg, $0.43 \mathrm{mmol}, 1.2$ equiv), S-Methyl-2-iodosulfoximine 2c (100 mg, $0.36 \mathrm{mmol}, 1$ equiv). After, $\mathrm{Et}_{3} \mathrm{~N}(73 \mu \mathrm{L}, 0.54$ $\mathrm{mmol}, 1.5$ equiv) was added drop by drop. Then, Dioxane $(1 \mathrm{~mL})$ was added and the mixture was stirred at $60^{\circ} \mathrm{C}$ for 2 days. The reaction mixture was cooled to room temperature and passed through a plug of celite and rinsed with EtOAc. This coupling product was purified by column chromatography on silica gel ((EtOAc: 100\%), and was isolated as a white solid in $49 \%$ yield as a mixture of two diastereoisomers. Both diastereoisomers $\mathbf{3} \mathbf{j a}$ and $\mathbf{3} \mathbf{j b}$ were separated through a preparative TLC (Cyclohexane/ EtOAc : 5/5).

\section{Diastereoisomer 3ja (amorphous)}

${ }^{1} \mathrm{H}$ NMR $\left(300 \mathrm{MHz}, \mathrm{CDCl}_{3}\right) \delta 8.14(\mathrm{~d}, J=7.8 \mathrm{~Hz}, 1 \mathrm{H}), 7.78(\mathrm{~d}, J=7.8 \mathrm{~Hz}$, $1 \mathrm{H}), 7.56$ (t, $J=7.5 \mathrm{~Hz}, 1 \mathrm{H}), 7.46(\mathrm{t}, J=7.5 \mathrm{~Hz}, 1 \mathrm{H}), 5.27(\mathrm{t}, J=8,7 \mathrm{~Hz}$, $1 \mathrm{H}), 5,19-5.06(\mathrm{~m}, 2 \mathrm{H}), 4.96(\mathrm{~d}, J=10.0 \mathrm{~Hz}, 1 \mathrm{H}), 4.21-4.07(\mathrm{~m}, 2 \mathrm{H})$ $3.78-3.72(\mathrm{~m}, 1 \mathrm{H}), 3.34(\mathrm{~s}, 3 \mathrm{H}), 2.85(\mathrm{bs}, \mathrm{NH}), 2.06-2.01(\mathrm{~m}, 12 \mathrm{H})$; ${ }^{13} \mathrm{C}$ NMR $\left(75 \mathrm{MHz}, \mathrm{CDCl}_{3}\right) \delta 170.52\left(\mathrm{C}_{\mathrm{q}}\right), 170.28\left(\mathrm{C}_{\mathrm{q}}\right), 169.48\left(\mathrm{C}_{q}\right)$, $169.23\left(\mathrm{C}_{\mathrm{q}}\right), 143.42\left(\mathrm{C}_{\mathrm{q}}\right), 134.10\left(\mathrm{C}_{\mathrm{q}}\right), 133.56(\mathrm{CH}), 133.17(\mathrm{CH}), 130.08$ $(\mathrm{CH}), 128.01(\mathrm{CH}), 85.95(\mathrm{CH}), 76.09(\mathrm{CH}), 73.93(\mathrm{CH}), 70.21(\mathrm{CH})$ $68.20(\mathrm{CH}), 62.11\left(\mathrm{CH}_{2}\right), 43.20\left(\mathrm{CH}_{3}\right), 20.83\left(\mathrm{CH}_{3}\right), 20.80\left(\mathrm{CH}_{3}\right), 20.70$ $\left(2 \times \mathrm{CH}_{3}\right) ; \mathrm{HRMS}(\mathrm{ESI}): \mathrm{m} / \mathrm{z}$ calcd for $\mathrm{C}_{21} \mathrm{H}_{28} \mathrm{~F}_{3} \mathrm{NO}_{10} \mathrm{~S}_{2}[\mathrm{M}+\mathrm{H}]+518.1151$; found 518.1155 .

\section{Compound 3jb (amorphous)}

${ }^{1} \mathrm{H}$ NMR $\left(300 \mathrm{MHz}, \mathrm{CDCl}_{3}\right) \delta 8.14(\mathrm{~d}, J=7.8 \mathrm{~Hz}, 1 \mathrm{H}), 7.77(\mathrm{~d}, J=7.8 \mathrm{~Hz}$, $1 \mathrm{H}), 7.55(\mathrm{t}, J=7.5 \mathrm{~Hz}, 1 \mathrm{H}), 7.45(\mathrm{t}, J=7.5 \mathrm{~Hz}, 1 \mathrm{H}), 5.27(\mathrm{t}, J=9.4,1 \mathrm{H})$, $5.19-5.07(\mathrm{~m}, 2 \mathrm{H}), 4.92(\mathrm{~d}, J=9.9 \mathrm{~Hz}, 1 \mathrm{H}), 4.24-4.08(\mathrm{~m}, 2 \mathrm{H}), 3.79-$ $3.75(\mathrm{~m}, 1 \mathrm{H}), 3.30(\mathrm{~s}, 3 \mathrm{H}), 2.51(\mathrm{bs}, \mathrm{NH}), 2.08-2.00(\mathrm{~m}, 12 \mathrm{H}) ;{ }^{13} \mathrm{C}$ NMR (75 MHz, $\mathbf{C D C l}_{3}$ ) ठ $170.53\left(\mathrm{C}_{\mathrm{q}}\right) 170.24\left(\mathrm{C}_{\mathrm{q}}\right), 169.49\left(\mathrm{C}_{\mathrm{q}}\right), 169.20\left(\mathrm{C}_{\mathrm{q}}\right)$, $143.29\left(\mathrm{C}_{\mathrm{q}}\right), 134.84\left(\mathrm{C}_{\mathrm{q}}\right), 133.57(\mathrm{CH}), 132.55(\mathrm{CH}), 130.06(\mathrm{CH}), 127.87$ $(\mathrm{CH}), 86.22(\mathrm{CH}), 76.01(\mathrm{CH}), 73.92(\mathrm{CH}), 70.33(\mathrm{CH}), 68.26(\mathrm{CH}), 62.27$ $\left(\mathrm{CH}_{2}\right), \quad 42.93\left(\mathrm{CH}_{3}\right), 20.83\left(\mathrm{CH}_{3}\right), 20.78\left(\mathrm{CH}_{3}\right), 20.71\left(2 \times \mathrm{CH}_{3}\right)$; HRMS(ESI): $\mathrm{m} / \mathrm{z}$ calcd for $\mathrm{C}_{21} \mathrm{H}_{28} \mathrm{~F}_{3} \mathrm{NO}_{10} \mathrm{~S}_{2}[\mathrm{M}+\mathrm{H}]+518.1151$; found 518.1155.

\section{$(2 R, 3 R, 4 S, 5 R, 6 S)-2$-(acetoxymethyl)-6-(((2R,3R,4S, 5R,6S)-4,5-} diacetoxy-2-(acetoxymethyl)-6-((2-) $(S$ -

(trifluoromethyl)sulfonimidoyl)phenyl)thio)tetrahydro-2H-pyran-3yl)oxy)tetrahydro-2H-pyran-3,4,5-triyl triacetate $\mathbf{3 k}$

A sealable tube purged with argon equipped with a cap was charged with $\mathrm{PdG}_{3}$-Xantphos (29 mg, $0.03 \mathrm{mmol}, 10 \mathrm{~mol} \%$ ), peracetylated Bthiocellobiose $\mathbf{1 i}(233 \mathrm{mg}, 0.36 \mathrm{mmol}, 1.2$ equiv), 2-iodo-sulfoximine $\mathbf{2 a}$ $(100 \mathrm{mg}, 0.3 \mathrm{mmol}, 1$ equiv). After, dioxane $(1 \mathrm{~mL})$ was added followed by adding $\mathrm{Et}_{3} \mathrm{~N}(61 \mu \mathrm{L}, 0.45 \mathrm{mmol}, 1.5$ equiv) dropwise. Then, the mixture was stirred at $60^{\circ} \mathrm{C}$ for $30 \mathrm{~min}$.

The reaction mixture was cooled to room temperature and passed through a plug of celite and rinsed with EtOAc. This crude was purified by column chromatography on silica gel (Cyclohexane/ EtOAc : 6/4), and compound 3k was isolated as a white solid in $60 \%$ yield. The ratio of diastereoisomers of compound $\mathbf{3 k}$ was about 1:0.93 respectively (by NMR).

The chromatographic separation was achieved using a Agilent infinity $1260(4.6 \times 150 \mathrm{~mm}) 5 \mu \mathrm{m}$ column. The mobile phase was Methanol and 
$\mathrm{H}_{2} \mathrm{O}+0.1 \mathrm{AcF}$ in the ratio of $55: 45, \mathrm{v} / \mathrm{v}$ in $30 \mathrm{~min}$. The column temperature was maintained at $25^{\circ} \mathrm{C}$ and the eluent was monitored at a wavelength of $254 \mathrm{~nm}$. The injection volume was $10 \mu \mathrm{L}$. The typical retention time of diastereoisomers of compound $\mathbf{3 k}$ was about $16.9 \mathrm{~min}$ and $20.9 \mathrm{~min}$, respectively.

\section{Diastereoisomer 3ka}

$\mathbf{R}_{\mathbf{f}}(40 \%$ EtOAc/Cyclohexane $)=0.5 ;[\boldsymbol{\alpha}]_{\mathbf{D}}{ }^{19}=-39.64\left(\mathrm{c}, 1.66\right.$ in $\left.\mathrm{CHCl}_{3}\right)$; mp: $226.1-228.5^{\circ} \mathbf{C}$; IR (neat, $\mathbf{c m}^{-1}$ ) 1756, 1452, 1259, 1035, $767 ;{ }^{1} \mathbf{H}$ NMR (300 MHz, $\left.\mathbf{C D C l}_{3}\right) \delta 8.26(\mathrm{~d}, J=7.8 \mathrm{~Hz}, 1 \mathrm{H}), 7.80(\mathrm{~d}, J=7.9 \mathrm{~Hz}$, $1 \mathrm{H}), 7.64$ (t, $J=7.7 \mathrm{~Hz}, 1 \mathrm{H}), 7.48$ (t, $J=7.7 \mathrm{~Hz}, 1 \mathrm{H}), 5.24$ (t, $J=8.9 \mathrm{~Hz}$, $1 \mathrm{H}), 5.17-5.03(\mathrm{~m}, 3 \mathrm{H}), 4.96-4.85(\mathrm{~m}, 2 \mathrm{H}), 4.53(\mathrm{~d}, J=8.2 \mathrm{~Hz}, 2 \mathrm{H})$, 4.36 (dd, $J=10.4,4.3 \mathrm{~Hz}, 1 \mathrm{H}), 4.18-4.03(\mathrm{~m}, 2 \mathrm{H}), 3.95(\mathrm{bs}, \mathrm{NH}), 3.81(\mathrm{t}$ $J=9.0 \mathrm{~Hz}, 1 \mathrm{H}), 3.75-3.62(\mathrm{~m}, 2 \mathrm{H}), 2.17-1.98(\mathrm{~m}, 21 \mathrm{H}),{ }^{13} \mathrm{C}$ NMR $(75$ $\left.\mathbf{M H z}, \mathbf{C D C l}_{3}\right) \delta 170.58\left(\mathrm{C}_{\mathrm{q}}\right), 170.31\left(\mathrm{C}_{\mathrm{q}}\right), 170.23\left(\mathrm{C}_{\mathrm{q}}\right), 169.83\left(\mathrm{C}_{\mathrm{q}}\right)$, $169.56\left(\mathrm{C}_{\mathrm{q}}\right), 169.42\left(\mathrm{C}_{\mathrm{q}}\right), 169.16\left(\mathrm{C}_{\mathrm{q}}\right), 138.94\left(\mathrm{C}_{\mathrm{q}}\right), 135.34(\mathrm{CH}), 134.00$ $(\mathrm{CH}), 132.40(\mathrm{CH}), 132.02\left(\mathrm{C}_{\mathrm{q}}\right), 127.62(\mathrm{CH}), 100.95(\mathrm{CH}), 85.09(\mathrm{CH})$, $76.98(\mathrm{CH}), 76.45(\mathrm{CH}), 73.72(\mathrm{CH}), 73.06(\mathrm{CH}), 72.24(\mathrm{CH}), 71.83(\mathrm{CH})$, $70.08(\mathrm{CH}), 67.97(\mathrm{CH}), 62.27\left(\mathrm{CH}_{2}\right), 61.74\left(\mathrm{CH}_{2}\right), 20.89\left(\mathrm{CH}_{3}\right), 20.78$ $\left(\mathrm{CH}_{3}\right), 20.66\left(5 \times \mathrm{CH}_{3}\right)$ (one carbon $\left(-\mathrm{CF}_{3}\right)$ is missing); ${ }^{19} \mathrm{~F}$ NMR (188 $\mathbf{~ M H z}$ $\mathrm{CDCl}_{3}$ ) $\delta$-76.42 (CF $)$; HRMS(ESI): $\mathrm{m} / \mathrm{z}$ calcd for $\mathrm{C}_{33} \mathrm{H}_{41} \mathrm{~F}_{3} \mathrm{NO}_{18} \mathrm{~S}_{2}[\mathrm{M}+\mathrm{H}]$ +860.1711 ; found 860.1697 . Aspect : White solid.

\section{Diastereoisomer $3 \mathrm{~kb}$}

$\mathbf{R}_{\mathbf{f}}(40 \%$ EtOAc/Cyclohexane $)=0.45 ;[\boldsymbol{\alpha}]_{\mathbf{D}}{ }^{19}=-50.06$ (c, 1.66 in $\left.\mathrm{CHCl}_{3}\right)$; mp: $227.2-229.8^{\circ} \mathbf{C}$; IR (neat, $\mathbf{~ c m}^{-1}$ ) 1746, 1453, 1291, 1032, $795 ;{ }^{1} \mathbf{H}$ NMR (300 MHz, CDCl $) \delta 8.18(\mathrm{~d}, J=7.8 \mathrm{~Hz}, 1 \mathrm{H}), 7.82(\mathrm{~d}, J=7.9 \mathrm{~Hz}$, $1 \mathrm{H}), 7.64(\mathrm{t}, J=7.4 \mathrm{~Hz}, 1 \mathrm{H}), 7.47(\mathrm{t}, J=7.6 \mathrm{~Hz}, 1 \mathrm{H}), 5.32-5.18(\mathrm{~m}, 1 \mathrm{H})$, $5.18-5.01(\mathrm{~m}, 3 \mathrm{H}), 4.93(\mathrm{t}, J=8.5 \mathrm{~Hz}, 1 \mathrm{H}), 4.78(\mathrm{~d}, J=10.1 \mathrm{~Hz}, 1 \mathrm{H})$, $4.55(\mathrm{t}, J=8.1 \mathrm{~Hz}, 2 \mathrm{H}), 4.36(\mathrm{dd}, J=12.5,4.2 \mathrm{~Hz}, 1 \mathrm{H}), 4.18-3.98(\mathrm{~m}$, $3 \mathrm{H}), 3.88-3.74(\mathrm{~m}, 2 \mathrm{H}), 3.72-3.62(\mathrm{~m}, 1 \mathrm{H}), 2.13-1.97(\mathrm{~m}, 21 \mathrm{H}) ;{ }^{13} \mathrm{C}$ NMR (75 MHz, $\left.\mathbf{C D C l}_{3}\right) \delta 170.57\left(\mathrm{C}_{\mathrm{q}}\right), 170.25\left(\mathrm{C}_{\mathrm{q}}\right), 169.79\left(\mathrm{C}_{\mathrm{q}}\right), 169.56$ $\left(\mathrm{C}_{\mathrm{q}}\right), 169.43\left(2 \times \mathrm{C}_{\mathrm{q}}\right), 169.18\left(\mathrm{C}_{\mathrm{q}}\right), 140.72\left(\mathrm{C}_{\mathrm{q}}\right), 135.57(\mathrm{CH}), 133.41(\mathrm{CH})$ $131.07(\mathrm{CH}), 127.14(\mathrm{CH}), 125.95\left(\mathrm{C}_{\mathrm{q}}\right), 101.00(\mathrm{CH}), 86.08(\mathrm{CH}), 76.83$ $(\mathrm{CH}), 76.53(\mathrm{CH}), 73.64(\mathrm{CH}), 73.09(\mathrm{CH}), 72.26(\mathrm{CH}), 71.85(\mathrm{CH}), 70.08$ $(\mathrm{CH}), 67.97(\mathrm{CH}), 62.41\left(\mathrm{CH}_{2}\right), 61.75\left(\mathrm{CH}_{2}\right), 20.92\left(\mathrm{CH}_{3}\right), 20.79\left(\mathrm{CH}_{3}\right)$, $20.67\left(4 \times \mathrm{CH}_{3}\right), 20.51\left(\mathrm{CH}_{3}\right)$ (one carbon $\left(-\mathrm{CF}_{3}\right)$ is missing); ${ }^{19} \mathrm{~F}$ NMR (188

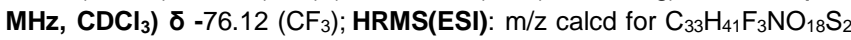
$[\mathrm{M}+\mathrm{H}]+860.1711$; found 860.1697. Aspect : White solid.

(2R,3R,4S,5R,6R)-2-(acetoxymethyl)-6-(((2R,3R,4S,5R,6R)-4,5diacetoxy-2-(acetoxymethyl)-6-(((2R,3R,4S,5R,6S)-4,5-diacetoxy-2 (acetoxymethyl)-6-((2-)(S-

(trifluoromethyl)sulfonimidoyl)phenyl)thio)tetrahydro-2H-pyran-3yl)oxy)tetrahydro-2H-pyran-3-yl)oxy)tetrahydro-2H-pyran-3,4,5-triyl triacetate 3I

A sealable tube purged with argon equipped with a cap was charged with $\mathrm{PdG}_{3}$-Xantphos (8 $\mathrm{mg}, 0.008 \mathrm{mmol}, 10 \mathrm{~mol} \%$ ), peracetylated Bthiomaltotriose $\mathbf{1 j}$ (150 $\mathrm{mg}, 0.16 \mathrm{mmol}, 2$ equiv), 2-iodo-sulfoximine 2a (26 mg, $0.08 \mathrm{mmol}, 1$ equiv). After, dioxane ( $1 \mathrm{~mL}$ ) was added followed by adding of $\mathrm{Et}_{3} \mathrm{~N}(16 \mu \mathrm{L}, 0.12 \mathrm{mmol}, 1.5$ equiv) dropwise. Then, the mixture was stirred at $60^{\circ} \mathrm{C}$ for $30 \mathrm{~min}$.

The reaction mixture was cooled to room temperature and passed through a plug of celite and rinsed with EtOAc. This crude was purified by column chromatography on silica gel (DCM/ $\mathrm{MeOH}$ : 95/5), and compound 3I was isolated as a colorless oil in $49 \%$ yield. The ratio of diastereoisomers of compound $\mathbf{3}$ I was about 1:0.73 (by NMR).

The chromatographic separation was achieved using an Agilent infinity $1260(4.6 \times 150 \mathrm{~mm}) 5 \mu \mathrm{m}$ column. The mobile phase was Acetonitrile and $\mathrm{H}_{2} \mathrm{O}+0.1 \mathrm{AcF}$ in the ratio of $50: 50, \mathrm{v} / \mathrm{v}$ in $25 \mathrm{~min}$. The column temperature was maintained at $25^{\circ} \mathrm{C}$ and the eluent was monitored at a wavelength of $254 \mathrm{~nm}$. The injection volume was $10 \mu \mathrm{L}$. The typical retention time of diastereoisomers of compound 3I was about 19.8 min and $21.2 \mathrm{~min}$, respectively.

\section{Diastereoisomer 3la}

$\mathbf{R}_{\mathbf{f}}(10 \% \mathrm{MeOH} / \mathrm{DCM})=0.6 ; \mathbf{\alpha}_{\mathbf{D}}{ }^{19}=-6.26\left(\mathrm{c}, 1.66\right.$ in $\left.\mathrm{CHCl}_{3}\right) ;$ IR (neat, $\mathbf{c m}^{-1}$ ) 1756, 1452, 1238, 1025, $767 ;{ }^{1} \mathbf{H}$ NMR (300 MHz, CDCl $\mathbf{~}_{3}$ ठ 8.27 (d, $J=7.3 \mathrm{~Hz}, 1 \mathrm{H}), 7.84(\mathrm{~d}, J=8.1 \mathrm{~Hz}, 1 \mathrm{H}), 7.68(\mathrm{t}, J=7.3 \mathrm{~Hz}, 1 \mathrm{H}), 7.49(\mathrm{t}$, $J=7.8 \mathrm{~Hz}, 1 \mathrm{H}), 5.44-5.29(\mathrm{~m}, 4 \mathrm{H}), 5.27(\mathrm{~d}, J=4.0 \mathrm{~Hz}, 1 \mathrm{H}), 5.06(\mathrm{t}, J=$ $9.0 \mathrm{~Hz}, 1 \mathrm{H}), 5.03-4.95(\mathrm{~m}, 2 \mathrm{H}), 4.86(\mathrm{dd}, J=10.5,3.9 \mathrm{~Hz}, 1 \mathrm{H}), 4.75(\mathrm{dd}$, $J=10.3,3.9 \mathrm{~Hz}, 1 \mathrm{H}), 4.53-4.42(\mathrm{~m}, 2 \mathrm{H}), 4.32(\mathrm{dd}, J=12.2,4.9 \mathrm{~Hz}, 1 \mathrm{H})$, $4.22(\mathrm{td}, J=12.8,3.6 \mathrm{~Hz}, 2 \mathrm{H}), 4.10-3.87(\mathrm{~m}, 6 \mathrm{H}), 3.87-3.77(\mathrm{~m}, 1 \mathrm{H})$, $2.15(\mathrm{~s}, 6 \mathrm{H}), 2.09(\mathrm{~s}, 3 \mathrm{H}), 2.07-1.92(\mathrm{~m}, 21 \mathrm{H}) ;{ }^{13} \mathrm{C}$ NMR (75 MHz, $\left.\mathbf{C D C l}_{3}\right)$ ठ $170.68\left(3 \times \mathrm{C}_{\mathrm{q}}\right), 170.48\left(\mathrm{C}_{\mathrm{q}}\right), 170.39\left(\mathrm{C}_{\mathrm{q}}\right), 170.14\left(\mathrm{C}_{\mathrm{q}}\right), 169.99$ $\left(\mathrm{C}_{\mathrm{q}}\right), 169.81\left(\mathrm{C}_{\mathrm{q}}\right), 169.67\left(\mathrm{C}_{\mathrm{q}}\right), 169.54\left(\mathrm{C}_{\mathrm{q}}\right), 138.86\left(\mathrm{C}_{\mathrm{q}}\right), 135.48(\mathrm{CH})$, $134.00(\mathrm{CH}), 132.39(\mathrm{CH}), 131.92\left(\mathrm{C}_{\mathrm{q}}\right), 127.64(\mathrm{CH}), 96.11(\mathrm{CH}), 95.89$ $(\mathrm{CH}), 84.78(\mathrm{CH}), 76.26(2 \times \mathrm{CH}), 74.04(\mathrm{CH}), 72.82(\mathrm{CH}), 71.87(\mathrm{CH})$, $70.64(\mathrm{CH}), 70.57(\mathrm{CH}), 70.25(\mathrm{CH}), 69.57(\mathrm{CH}), 69.27(\mathrm{CH}), 68.74(\mathrm{CH})$, $68.14(\mathrm{CH}), 63.30\left(\mathrm{CH}_{2}\right), 62.59\left(\mathrm{CH}_{2}\right), 61.61\left(\mathrm{CH}_{2}\right), 20.99\left(\mathrm{CH}_{3}\right), 20.93$ $\left(2 \times \mathrm{CH}_{3}\right), 20.79\left(\mathrm{CH}_{3}\right), 20.71\left(5 \times \mathrm{CH}_{3}\right), 20.61\left(\mathrm{CH}_{3}\right)$, (one carbon $\left(-\mathrm{CF}_{3}\right)$ is missing); ${ }^{19} \mathrm{~F}$ NMR (188 MHz, $\mathrm{CDCl}_{3}$ ) $\delta$-76.46 ( $\left.\mathrm{CF}_{3}\right)$; HRMS(ESI): m/z calcd for $\mathrm{C}_{45} \mathrm{H}_{57} \mathrm{~F}_{3} \mathrm{NO}_{26} \mathrm{~S}_{2}[\mathrm{M}+\mathrm{H}]+1148.2556$; found 1148.2462. Aspect : Colorless oil.

\section{Diastereoisomer 3Ib}

$\mathbf{R}_{\mathbf{f}}(10 \% \mathrm{MeOH} / \mathrm{DCM})=0.51 ;[\boldsymbol{\alpha}]_{\mathrm{D}}{ }^{19}=+56.32\left(\mathrm{c}, 1.66\right.$ in $\left.\mathrm{CHCl}_{3}\right)$; IR (neat, $\left.\mathbf{c m}^{-1}\right)$ 1739, 1368, 1212, 1027, 735; ${ }^{1} \mathbf{H}$ NMR (300 MHz, $\left.\mathbf{C D C l}_{3}\right) \mathbf{\delta} 8.18$ (d, $J=8.0 \mathrm{~Hz}, 1 \mathrm{H}), 7.86(\mathrm{~d}, J=7.8 \mathrm{~Hz}, 1 \mathrm{H}), 7.68(\mathrm{t}, J=7.2 \mathrm{~Hz}, 1 \mathrm{H}), 7.48(\mathrm{t}$, $J=7.5 \mathrm{~Hz}, 1 \mathrm{H}), 5.43-5.24(\mathrm{~m}, 5 \mathrm{H}), 5.06(\mathrm{t}, J=9.9 \mathrm{~Hz}, 1 \mathrm{H}), 4.98(\mathrm{t}, J=$ $9.0 \mathrm{~Hz}, 1 \mathrm{H}), 4.90-4.82(\mathrm{~m}, 2 \mathrm{H}), 4.75(\mathrm{dd}, J=8.4,4.0 \mathrm{~Hz}, 1 \mathrm{H}), 4.54-$ $4.42(\mathrm{~m}, 2 \mathrm{H}), 4.35(\mathrm{dd}, J=9.0,5.2 \mathrm{~Hz}, 1 \mathrm{H}), 4.23(\mathrm{td}, J=12.0,3.6 \mathrm{~Hz}$, 2H), $4.12-3.83(\mathrm{~m}, 7 \mathrm{H}), 2.17(\mathrm{~s}, 3 \mathrm{H}), 2.16(\mathrm{~s}, 3 \mathrm{H}), 2.09(\mathrm{~s}, 3 \mathrm{H}), 2.05-$ $1.98(\mathrm{~m}, 21 \mathrm{H}) ;{ }^{13} \mathbf{C}$ NMR (75 MHz, $\left.\mathbf{C D C l}_{3}\right) \delta 170.50\left(3 \times \mathrm{C}_{\mathrm{q}}\right), 170.32\left(\mathrm{C}_{\mathrm{q}}\right)$, $170.23\left(\mathrm{C}_{\mathrm{q}}\right), 169.92\left(\mathrm{C}_{\mathrm{q}}\right), 169.82\left(\mathrm{C}_{\mathrm{q}}\right), 169.65\left(\mathrm{C}_{\mathrm{q}}\right), 169.47\left(\mathrm{C}_{\mathrm{q}}\right), 169.37$ $\left(\mathrm{C}_{\mathrm{q}}\right), 140.54\left(\mathrm{C}_{\mathrm{q}}\right), 135.55(\mathrm{CH}), 133.22(\mathrm{CH}), 130.82(\mathrm{CH}), 126.94(\mathrm{CH})$, $122.88\left(\mathrm{C}_{\mathrm{q}}\right), 95.93(\mathrm{CH}), 95.73(\mathrm{CH}), 85.58(\mathrm{CH}), 76.07(\mathrm{CH}), 75.92(\mathrm{CH})$, $73.92(\mathrm{CH}), 72.71(\mathrm{CH}), 71.70(\mathrm{CH}), 70.41(\mathrm{CH}), 70.08(2 \times \mathrm{CH}), 69.41$ $(\mathrm{CH}), 69.11(\mathrm{CH}), 68.58(\mathrm{CH}), 67.97(\mathrm{CH}), 63.29\left(\mathrm{CH}_{2}\right), 62.47\left(\mathrm{CH}_{2}\right)$, $61.44\left(\mathrm{CH}_{2}\right), 20.81-20.32\left(10 \times \mathrm{CH}_{3}\right)$, (one carbon $\mathrm{Cq}$ is missing); ${ }^{19} \mathbf{F}$ NMR (188 $\left.\mathbf{M H z}, \mathrm{CDCl}_{3}\right) \quad \delta \quad-76.11 \quad\left(\mathrm{CF}_{3}\right) ; \mathrm{HRMS}(\mathrm{ESI}): \mathrm{m} / \mathrm{z}$ calcd for $\mathrm{C}_{45} \mathrm{H}_{57} \mathrm{~F}_{3} \mathrm{NO}_{26} \mathrm{~S}_{2}[\mathrm{M}+\mathrm{H}]+1148.2556$; found 1148.2462. Aspect: Colorless oil.

\section{Coupling of enantiopure sulfoximines 2aa, 2ab}

(2R,3S, 4S,5R,6S)-2-(acetoxymethyl)-6-((2-((S)-imino(I1oxidanyl)(trifluoromethyl)-I5-sulfanyl)phenyl)thio)tetrahydro-2H-pyran3,4,5-triyl triacetate 3aa'

A sealable tube purged with argon equipped with a cap was charged with $\mathrm{PdG}_{3}$-Xantphos (9 mg, $0.009 \mathrm{mmol}, 10 \mathrm{~mol} \%$ ), tetraacetylated $B$ thiogalactose 1a (49 mg, $0.13 \mathrm{mmol}, 1.5$ equiv), (R)-(+)-2-iodosulfoximine 2aa (30 mg, $0.09 \mathrm{mmol}, 1$ equiv). Then, Dioxane ( $1 \mathrm{~mL})$ was added and to the solution was added $\mathrm{Et}_{3} \mathrm{~N}$ (19 $\mu \mathrm{L}, 0.13 \mathrm{mmol}, 1.5$ equiv) dropwise and the mixture was stirred at $60^{\circ} \mathrm{C}$ for $30 \mathrm{~min}$.

The reaction mixture was cooled to room temperature and passed through a plug of celite and rinsed with EtOAc. This coupling product was purified by column chromatography on silica gel (Pentane/ EtOAc: 5/5), and 3aa'was isolated as a white solid in $78 \%$ yield.

$\mathbf{R}_{\mathbf{f}}(50 \%$ EtOAc/Pentane $)=0.57 ;[\boldsymbol{\alpha}]_{\mathbf{D}}{ }^{19}=+8.37$ (c, 1.66 in $\left.\mathrm{CHCl}_{3}\right) ; \mathbf{m p}$ $50.3-54.0^{\circ} \mathbf{C}$; IR (neat, $\mathbf{c m}^{-1}$ ) 1749, 1368, 1213, 1174, 1083, $1058 ;{ }^{1} \mathbf{H}$ NMR (300 MHz, $\left.\mathbf{C D C l}_{3}\right) \delta 8.25(\mathrm{~d}, J=8.0 \mathrm{~Hz}, 1 \mathrm{H}), 7.91(\mathrm{~d}, J=8.0 \mathrm{~Hz}$, $1 \mathrm{H}), 7.64(\mathrm{t}, J=7.7 \mathrm{~Hz}, 1 \mathrm{H}), 7.48(\mathrm{t}, J=7.7 \mathrm{~Hz}, 1 \mathrm{H}), 5.46(\mathrm{~d}, J=3.2 \mathrm{~Hz}$, 1H), 5.39 (t, $J=9.9 \mathrm{~Hz}, 1 \mathrm{H}), 5.09(\mathrm{dd}, J=9.9,3.2 \mathrm{~Hz}, 1 \mathrm{H}), 4.89(\mathrm{~d}, J=$ 
$10.1 \mathrm{~Hz}, 1 \mathrm{H}), 4.18-4.15(\mathrm{~m}, 2 \mathrm{H}), 4.04-3.97(\mathrm{~m}, 2 \mathrm{H}), 2.17(\mathrm{~s}, 3 \mathrm{H}), 2.03$ $(\mathrm{s}, 6 \mathrm{H}), 1.97(\mathrm{~s}, 3 \mathrm{H}) ;{ }^{13} \mathrm{C}$ NMR $\left(75 \mathrm{MHz}, \mathrm{CDCl}_{3}\right) \delta 170.36\left(\mathrm{C}_{\mathrm{q}}\right), 170.16$ $\left(\mathrm{C}_{\mathrm{q}}\right), 160.06\left(\mathrm{C}_{\mathrm{q}}\right), 169.39\left(\mathrm{C}_{\mathrm{q}}\right), 138.99\left(\mathrm{C}_{\mathrm{q}}\right), 135.22(\mathrm{CH}), 133.96(\mathrm{CH})$, $132.45(\mathrm{CH}), 131.83\left(\mathrm{C}_{\mathrm{q}}\right), 127.52(\mathrm{CH}), 121.16\left(\mathrm{CF}_{3}, \mathrm{q}, \mathrm{J}_{\mathrm{CF}}=333 \mathrm{~Hz}\right)$, $85.60(\mathrm{CH}), 74.81(\mathrm{CH}), 72.08(\mathrm{CH}), 67.34(\mathrm{CH}), 66.67(\mathrm{CH}), 61.84\left(\mathrm{CH}_{2}\right)$, $20.74\left(2 \times \mathrm{CH}_{3}\right), 20.63\left(2 \times \mathrm{xH}_{3}\right) ;{ }^{19} \mathrm{~F}$ NMR $\left(188 \mathrm{MHz}, \mathrm{CDCl}_{3}\right) \delta-76.55$ $\left(\mathrm{CF}_{3}\right) ; \mathrm{HRMS}(\mathrm{ESI}): \mathrm{m} / \mathrm{z}$ calcd for $\mathrm{C}_{21} \mathrm{H}_{25} \mathrm{~F}_{3} \mathrm{NO}_{10} \mathrm{~S}_{2}[\mathrm{M}+\mathrm{H}]+572.0866$; found 1148.0864. Aspect : White solid.

\section{$(2 R, 3 S, 4 S, 5 R, 6 S)-2-($ acetoxymethyl)-6-((2-)((R)-imino(11-} oxidanyl)(trifluoromethyl)-15-sulfanyl)phenyl)thio)tetrahydro-2H-pyran3,4,5-triyl triacetate $3 \mathbf{a b}^{\prime}$

A sealable tube purged with argon equipped with a cap was charged with $\mathrm{PdG}_{3}$-Xantphos $(9 \mathrm{mg}, 0.009 \mathrm{mmol}, 10 \mathrm{~mol} \%$ ), tetraacetylated Bthiogalactose 1a (49 mg, $0.13 \mathrm{mmol}, 1.5$ equiv), (S)-(-)-2-iodosulfoximine $2 \mathrm{ab}$ ( $30 \mathrm{mg}, 0.09 \mathrm{mmol}, 1$ equiv). Then, Dioxane $(1 \mathrm{~mL})$ was added and to the solution was added $\mathrm{Et}_{3} \mathrm{~N}(19 \mu \mathrm{L}, 0.13 \mathrm{mmol}, 1.5$ equiv) dropwise and the mixture was stirred at $60^{\circ} \mathrm{C}$ for $30 \mathrm{~min}$.

The reaction mixture was cooled to room temperature and passed through a plug of celite and rinsed with EtOAc. This coupling product was purified by column chromatography on silica gel (Pentane/ EtOAc: 5/5), and $\mathbf{3 a b}$ ' was isolated as a white solid in $84 \%$ yield.

$\mathbf{R}_{\mathbf{f}}(50 \%$ EtOAc/Pentane $)=0.5 ;[\boldsymbol{\alpha}]_{\mathbf{D}}{ }^{19}=+83.43\left(\mathrm{c}, 1.66\right.$ in $\left.\mathrm{CHCl}_{3}\right) ; \mathbf{m p}:$ $48.0-52.0^{\circ} \mathbf{C}$; IR (neat, $\mathbf{c m}^{-1}$ ) $1760,1367,1243,1085,1069 ;{ }^{1} \mathbf{H}$ NMR $\left(300 \mathrm{MHz}, \mathrm{CDCl}_{3}\right) \delta 8.17(\mathrm{~d}, J=7.9 \mathrm{~Hz}, 1 \mathrm{H}), 7.83(\mathrm{~d}, J=8.0 \mathrm{~Hz}, 1 \mathrm{H})$, 7.63 (t, $J=7.7 \mathrm{~Hz}, 1 \mathrm{H}), 7.45$ (t, $J=7.7 \mathrm{~Hz}, 1 \mathrm{H}), 5.46(\mathrm{~d}, J=3.2 \mathrm{~Hz}, 1 \mathrm{H})$, 5.35 (t, $J=10.0 \mathrm{~Hz}, 1 \mathrm{H}), 5.08(\mathrm{dd}, J=9.9,3.2 \mathrm{~Hz}, 1 \mathrm{H}), 4.80(\mathrm{~d}, J=10.1$ $\mathrm{Hz}, 1 \mathrm{H}), 4.21-4.07(\mathrm{~m}, 3 \mathrm{H}), 4.07-4.02(\mathrm{~m}, 1 \mathrm{H}), 2.16(\mathrm{~s}, 3 \mathrm{H}), 2.05(\mathrm{~s}$, 3H), 2.01 (s, 3H), $1.97(\mathrm{~s}, 3 \mathrm{H}) ;{ }^{13} \mathrm{C}$ NMR $\left(75 \mathrm{MHz}, \mathrm{CDCl}_{3}\right) \delta 170.33\left(\mathrm{C}_{\mathrm{q}}\right)$, $170.24\left(\mathrm{C}_{\mathrm{q}}\right), 170.03\left(\mathrm{C}_{\mathrm{q}}\right), 169.44\left(\mathrm{C}_{\mathrm{q}}\right), 140.70\left(\mathrm{C}_{\mathrm{q}}\right), 135.51(\mathrm{CH}), 133.42$ $(\mathrm{CH}), 131.14(\mathrm{CH}), 127.98\left(\mathrm{C}_{\mathrm{q}}\right), 127.07(\mathrm{CH}), 121.33\left(\mathrm{q}, \mathrm{J}_{\mathrm{CF}}=334 \mathrm{~Hz}\right.$, $\left.\mathrm{CF}_{3}\right), 86.49(\mathrm{CH}), 74.63(\mathrm{CH}), 71.97(\mathrm{CH}), 67.26(\mathrm{CH}), 66.80(\mathrm{CH}), 61.99$ $\left(\mathrm{CH}_{2}\right), 20.73\left(2 \times \mathrm{CH}_{3}\right), 20.62\left(\mathrm{CH}_{3}\right), 20.53\left(\mathrm{CH}_{3}\right) ;{ }^{19} \mathrm{~F}$ NMR (188 MHz, $\left.\mathrm{CDCl}_{3}\right)$ ठ $-76.25\left(\mathrm{CF}_{3}\right) ; \mathrm{HRMS}(\mathrm{ESI}): \mathrm{m} / \mathrm{z}$ calcd for $\mathrm{C}_{21} \mathrm{H}_{25} \mathrm{~F}_{3} \mathrm{NO}_{10} \mathrm{~S}_{2}[\mathrm{M}+\mathrm{H}]$ +572.0866 ; found 1148.0866. Aspect : White solid.

\section{Acknowledgements ((optional))}

Paris-Sud, ANR (ANR-15-CE29- 0002), and by la Ligue Contre le Cancer through an Equipe Labellisée 2014 grant. We thank N. Vanthuyne (iSm2, Marseille) for some preparative HPLC. We also thank the Algerian Ministry of Education and Research for a fellowship (Profas B+) to Nedjwa Bennai. Our laboratory is a member of the Laboratory of Excellence LERMIT supported by a grant (ANR-10-LABX-33). GIS Fluor (French Fluorine Network) is also acknowledged.

Keywords: S-trifluoromethyl; S-arylsulfoximines; thioglycosides; Palladium; Migita Cross-Couling

[1] a) U. Lücking, Angew. Chem. Int. Ed. 2013, 52, 9399-9 408; b) U. Lücking, Org. Chem. Front. 2019, 6, 1319-1324; c) M. Frings, C. Bolm, A. Blum, C. Gnamm, Eur. J. Med. Chem. 2017, 126, 225-245.

[2] J. A. Sirvent, U. Lücking, ChemMedChem, 2017, 12, 487-501.

[3] T. C. Sparks, G. B. Watson, M. R. Loso, C. Geng, J. M. Babcock, J. D. Thomas, Pestic. Biochem. Physiol. 2013, 107, 1-7.

[4] a) J. Wang, M. Sánchez-Roselló, J. L. Aceňa, C. del Pozo, A. E. Sorochinsky, S. Fustero, V. A. Soloshonok, H. Liu, Chem. Rev. 2014, 114, 2432-; b) H. Mei, J. Han, S. Fustero, M.Medio-Simon, D. M. Sedwick, C. Santi, R. Ruzziconi, V. A. Soloshonok, Chem. Eur. J. 2019, 25, 11797-11819; c) E. A. Ilardi, E. Vitaku, J. T. Njardarson, J. Med.
Chem. 2014, 57, 2832-2842; d) D. O'Hagan, J. Fluorine Chem. 2010 131, 1071-1081; (e) T. Fujiwara, D. J. O'Hagan, J. Fluorine Chem. 2014, 167, 16-29.

[5] For reviews see: a) V. Bizet, R. Kowalczyk, C. Bolm, Chem. Soc. Rev. 2014, 43, 2426-2438; b) X. Shen, J. Hu, Eur. J. Org. Chem. 2014 4437-4451; c) A.-L. Barthelemy, E. Magnier, C. R. Chimie. 2018, 21, 711-722.

[6] N. Nishimura, M. H. Norman, L. Liu, K. C.; Yang, K. S. Ashton, M. D. Bartberger, S. Chmait, J. Chen, R. Cupples, C. Fotsch, J. Helmering, S. R. Jordan, R. K. Kunz, L. D. Pennington, S. F. Poon, A. Siegmund, G. Sivits, D. J. Lloyd, C. Hale, D. J. St. Jean, J. Med. Chem. 2014, 57, 3094-3116.

[7] a) For general reviews, see: a) H. Driguez, Top. Curr. Chem., 1997, 187, 85-116; b) H. Driguez, ChemBioChem, 2001, 2, 311-318; c) K. Pachamuthu and R. R. Schmidt, Chem. Rev., 2006, 106, 160-187; d) M. Samuni-Blank, I. Izhaki, M. D. Dearing, Y. Gerchman, B. Trabelcy, A L. W. H. Karasov, Z. Arad, Curr. Biol. 2012, 22, 1218-1220; e) G. Lian, X. Zhang and B. Yu, Carbohydr. Res., 2015, 403, 13-22. For Selected articles: f) F. Castaneda, A. Burse, W. Boland, R. K.-H. Kinne, Int. J. Med. Sci. 2007, 4, 131-139.; g) J. Rodrigue, G. Ganne, B. Blanchard, C. Saucier, D. Giguere, T. C. Shiao, A. Varrot, A. Imberty, R. Roy, Org. Biomol. Chem. 2013, 11, 6906-6918; h) G. H. Elgemeie, A. B. Farag, K M. Amin, O. M. El-Badry, G. S. Hassan, Med. Chem. 2014, 4, 814-820; i) M. R. Aouad, Nucleos. Nucleot. Nucl. Acids, 2016, 35, 1-15; j) E. Kato, H. Nagano, S. Yamamura, M. Ueda, Tetrahedron, 2003, 59, 5909-5917; k) M. Schnabelrauch, A. Vasella, S. G. Withers, Helv. Chim. Acta. 1994, 77, 778-799; I) M. Adinolfi, M. d'Ischia, A. ladonisi, L. Leone, A. Pezzella, S. Valerio, J. Org. Chem. 2012, 23, 4333-4338; m) K. Shirota, Y. Kato, K. Suzuki, Y. Sugiyama, J. Pharmacol. Exp. Ther 2001, 299, 459-467; n) R. Caraballo, M. Sakulsombat, O. Ramstrçm, ChemBioChem 2010, 11, 1600-1606

[8] a) C. S. Rye, S. G. Withers, Carbohydr. Res. 2004, 339, 699-703; b) B. B. Metaferia, B. J. Fetterolf, S. Shazad-ul-Hussan, M. Moravec, J. A Smith, S. Ray, M.-T. Gutierrez-Lugo, C. A. Bewley, J. Med. Chem. 2007, 50, 6326-6336.

[9] a) J. D. C. Codee, R. E. J. N. Litjens, L. J. van den Bos, H. S Overkleeft, G. A. van der Marel, Chem. Soc. Rev. 2005, 34, 769-782 for selected examples, see: b) M. Johannes, M. Reindl, B. Gerlitzki, E. Schmitt, A. Hoffmann-Rçder, Beilstein J. Org. Chem. 2015, 11, 155161; c) D. Budhadev, B. Mukhopadhyay, Carbohydr. Res. 2014, 384, 51-55; d) N. Basu, S. Kumar Maity, R. Ghosh, RSC Adv. 2012, 2, 12661-12664; e) P. Verma, R. Raj, B. Roy, Mukhopadhyay, B. Tetrahedron: Asymmetry 2010, 21, 2413-2418; f) D. Xiong, C.; Zhang, L. H.; Ye, X. S. Adv. Synth. Catal. 2008, 350, 1696-1700; g) B. Roy, K. Pramanik, B. Mukhopadhyay, Glycoconjugate J. 2008, 25, 157-166; h) Y. Zeng, Z. Wang, D. Whitfield, X. Huang, J. Org. Chem. 2008, 73, 7952-7579; i) M. Fridman, V. Belakhov, L. V. Lee, F.-S. Liang, C.-H. Wong, T. Baasov, Angew. Chem. Int. Ed. 2005, 44, 447-452.

[10] a) N. A. Meanwell, J. Med. Chem. 2011, 54, 2529-2591; b) D. Lu, R. Vince, Bioorg. Med. Chem. Lett. 2007, 17, 5614-5619.

[11] a) Y. Mace, C. Urban, C. Pradet, J. Marrot, J.-C. Blazejewski, E. Magnier, Eur. J. Org. Chem. 2009, 3150-3153; b) S. Chaabouni, J.-F. Lohier, A.-L. Barthelemy, T. Glachet, E. Anselmi, G. Dagousset, P. Diter, B. Pégot, E. Magnier, V. Reboul Chem. Eur. J. 2018, 64, 1700617010.

[12] For a recent review, see: N. Ibrahim, M. Alami, S. Messaoudi, Asian. J. Org. Chem. 2018, 7, 2026-2038. For selected examples, see: a) A. Chabrier, A. Bruneau, S. Benmahdjoub, B. Benmerad, S. Belaid, J.-D Brion, M. Alami, S. Messaoudi, Chem.-Eur. J., 2016, 22, 15006-15010; b) D. Montoir, M. Amoura, Z. E.-A. Ababsa, T. M. Vishwanath, E. YenPon, V. Robert, M. Beltramo, V. Piller, M. Alami, A. Aucagne, S. Messaoudi, Chem. Sci., 2018, 9, 8753-8759; c) S. Benmahdjoub, N Ibrahim, B. Benmerad, M. Alami, S. Messaoudi, Org. Lett. 2018, 20, 4067-4071; d) M. Zhu, G. Dagousset, M. Alami, E. Magnier, S. Messaoudi. Org. Lett. 2019, 21, 5132-5137.

[13] a) T. Migita, T. Shimizu, Y. Asami, J.-I. Shiobara, Y. Kato, M. Kosugi. Bull. Chem. Soc. Jpn. 1980, 53, 1385-1389; b) M. Kosugi, T. Shimizu, T. Migita. Chem. Lett. 1978, 7, 13-14. 
[14] T.-N. Le, P. Diter, B. Pegot, C. Bournaud, M. Toffano, R. Guillot, G. VoThanh, E. Magnier, Org. Lett. 2016, 18, 5102-5105.

[15] A.-L. Barthelemy, E. Anselmi, T.-N. Le, G. Vo-Thanh, R. Guillot, K. Miqueu, E. Magnier, J. Org. Chem. 2019, 84, 4086-4094.

[16] a) T.-N. Le, E. Kolodziej, P. Diter, B. Pégot, C. Bournaud, M. Toffano, R. Guillot, G. Vo-Thanh, E. Magnier, Chimia 2014, 68, 410-413; b) J. Kalim, T. Duhail, T.-H. Le, N. Vanthuyne, E. Anselmi, A. Togni, E. Magnier, Chem. Sci. 2019, 10, 10516-10523.

[17] A. Bruneau, M. Roche, A. Hamze, J. -D. Brion, M. Alami, S. Messaoudi. Chem. Eur. J. 2015, 21, 8375-8379.

[18] N. C. Bruno, M. T. Tudge, S. L. Buchwald. Chem. Sci., 2013, 4, 916920 
